\title{
Electrified cone formation in perfectly conducting viscous liquids: Self-similar growth irrespective of Reynolds number
}

Cite as: Phys. Fluids 31, 102103 (2019); https://doi.org/10.1063/1.5123742

Submitted: 08 August 2019 . Accepted: 18 September 2019 . Published Online: 14 October 2019

Theodore G. Albertson, and Sandra M. Troian

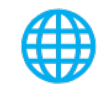

AIP Author Services English Language Editing 


\title{
Electrified cone formation in perfectly conducting viscous liquids: Self-similar growth irrespective of Reynolds number
}

\author{
Cite as: Phys. Fluids 31, 102103 (2019); doi: 10.1063/1.5123742 \\ Submitted: 8 August 2019 - Accepted: 18 September 2019 • \\ Published Online: 14 October 2019
}

Theodore G. Albertson and Sandra M. Troian

\begin{abstract}
AFFILIATIONS
California Institute of Technology, T. J. Watson Sr. Laboratories of Applied Physics, 1200 E. California Blvd., MC 128-95, Pasadena, California 91125, USA
\end{abstract}

a) Author to whom correspondence should be addressed: stroian@caltech.edu. URL: www.troian.caltech.edu.

\begin{abstract}
Above a critical field strength, the free surface of an electrified, perfectly conducting viscous liquid, such as a liquid metal, is known to develop an accelerating protrusion resembling a cusp with a conic tip. Field self-enhancement from tip sharpening is reported to generate divergent power law growth in finite time of the forces acting in that region. Previous studies have established that tip sharpening proceeds via a selfsimilar process in two distinguished limits-the Stokes regime and the inviscid regime. Using finite element simulations to track the shape and forces acting at the tip of an electrified protrusion in a perfectly conducting Newtonian liquid, we demonstrate that the conic tip always undergoes self-similar growth irrespective of the Reynolds number. The blowup exponents at the conic apex for all terms in the NavierStokes equation and the normal stress boundary condition at the moving interface reveal the dominant forces at play as the Reynolds number increases. Rescaling of the tip shape by the power law representing the divergence in capillary stress at the apex yields an excellent collapse onto a universal cone shape with an interior half-angle dependent on the Maxwell stress. The rapid acceleration of the liquid interface also generates a thin interfacial boundary layer characterized by a significant rate of strain. Additional details of the modeled flow, applicable to cone growth in systems such as liquid metal ion sources, help dispel prevailing misconceptions that dynamic cones resemble conventional Taylor cones or that viscous stresses at a finite Reynolds number can be neglected.
\end{abstract}

Published under license by AIP Publishing. https://doi.org/10.1063/1.5123742

\section{INTRODUCTION}

In a short influential paper in 1884, Lord Rayleigh demonstrated with a simple argument based on linear stability analysis why a charged spherical mass of liquid acted upon solely by electrical and capillary forces represents a state of "unstable equilibrium.” Rayleigh's hydrodynamic analysis was especially insightful given that Maxwell's equations had only been developed a couple of decades earlier. Rayleigh also predicted that the charge instability would cause the spherical mass to distort into a peaked structure that should emit a fine jet due to the growing imbalance between the destabilizing electrical pressure and the stabilizing capillary pressure. Several years later in 1890, Larmor also became interested in the electrification of capillary waves on a liquid surface. ${ }^{1}$ Seemingly unaware of Rayleigh's work, he examined the linear stability of an electrified liquid surface by appealing to the unsteady form of
Bernoulli's equation. He derived the dispersion relation correlating the rate of growth to the wavelength of ripples along the interface of a charged liquid film. These two early studies revealed why electrified liquid surfaces can sprout and grow slender protrusions, which narrow and sharpen in time.

\section{A. Instability analyses by Tonks and Frenkel}

This phenomenon remained a curiosity for decades until 1935 when Tonks, ${ }^{2}$ a researcher at General Electric who was investigating ion emission from solid metal electrodes, turned his attention to sparking and charge emission in liquid metals. Tonks closely examined the liquid distortion process and subsequent electrical breakdown of a liquid mercury surface held in close proximity to a counter electrode subject to field strengths approaching several megavolts per centimeter. He noted that above a critical field strength, a planar 
liquid metal would rapidly generate an accelerating protrusion that narrowed in time, thereby concentrating the local disruptive electric field about a liquid cusp that eventually underwent ion emission. His studies confirmed that field induced ion emission from liquid metals occurred at a lower field strength than for solid metals. To complement these observations, he carried out a number of calculations in the inviscid limit that resulted in equations and estimates relating the amplitude of the distortion of an electrified liquid surface to the minimal field strength required to induce a sustained deformation, as well as the time required for interface rupture to give way to ion emission. All along, Tonks emphasized two questions that drove forward his work-namely, what is the relation between the applied field strength, the distortion amplitude, and the pressure at the tip of an electrified accelerating protrusion and how fast does this protrusion accelerate in response to the fluid pressure at the tip. Realizing that a complete rigorous solution to this electrohydrodynamic problem was too complicated, he instead developed a perceptive approximate treatment that revealed critical aspects of the liquid runaway distortion process. Shortly after Tonks published this work, Frenkel ${ }^{3}$ provided a more general derivation of the liquid distortion process for inviscid fluids by solving the equation for the velocity of surface waves triggered by an applied electric field. The periodic distortion of the surface of a perfectly conducting liquid above a critical field strength is now known as the Larmor-TonksFrenkel instability. Despite the growing body of work on this subject during the past century, the two questions originally posed by Tonks in 1935 have not yet been completely answered, partly due to the fact that viscous effects significantly complicate the analysis for flow at a finite Reynolds number.

The investigations by Larmor and Frenkel relied on a stability analysis of electrified capillary waves on a perfectly conducting inviscid liquid, from which it was found that the wave frequency becomes complex valued once the critical field strength for sustained distortion is exceeded. By contrast, Tonks focused on the dynamic evolution of a single protrusion emanating from a flat liquid surface with an initial small bump modeled as a spherical cap. His analysis revealed that beyond a critical field strength, the pressure at the conic apex cannot support equilibrium since the electric pressureknown as the Maxwell pressure or Maxwell stress-increases as the square of the apex height. This quadratic dependence cannot be balanced by the restoring hydrostatic or capillary pressure, which at best scales linearly or inversely with apex height, respectively. Therefore, any small increase in the apex height simply increases the net local unbalanced pressure. This strong imbalance causes a runaway process by which the distortion progressively increases in height. By relying on various approximations for the initial conditions and simplified functional forms for other quantities, Tonks was successful in deriving an equation for the acceleration of an eccentric liquid shape with time. Remarkably, Tonks also appears to have realized that the growth of the liquid tip proceeds in self-similar fashion, as seems apparent from his hand drawn sketch in Fig. 8 of Ref. 2 showing snapshots of the evolution of an electrified liquid tip.

\section{B. Experimental studies by Krohn and co-workers}

Interest in this phenomenon began spreading rapidly and by the early 1960s, Krohn ${ }^{4}$ and co-workers at TRW recognized that the spontaneous process by which an electrified liquid can undergo fission to produce charged droplets and ions could be of enormous relevance to the burgeoning field of electrostatic propulsion ${ }^{5-8}$ for "space thrusters." Also, while other research groups focused on colloid thrusters using electrospray propulsion from dielectric liquids, Krohn instead concentrated on cusp formation in liquid alkali metals. Within just a few years, he was able to demonstrate sustained ion emission from small liquid droplets of cesium, gallium, and mercury placed on the tip of a tungsten needle held at a potential of a few kilovolts. By 1975, Krohn successfully demonstrated stable emission lasting several hours, an exceptional technological achievement which paved the way for liquid metal ion sources (LMISs). By the late 1970s, researchers ${ }^{9,10}$ recognized the potential of LMIS for high-resolution ion microprobe analysis, micromilling, and microimplantation. Attention soon turned to issues involving control of operational parameters affecting the diameter of the ion source area, the maximum brightness achievable, and the energy spread of the emitted ion beam. Significant industrial support during this period led to rapid and far reaching development of this field, so much so that LMIS now forms the basis for instrumentation ranging from high resolution focused ion beam systems for enhanced etching and deposition to scanning ion microscopy, micromilling, ion mass spectrometry, implantation, and lithography. ${ }^{11}$ Currently, the field is moving toward miniaturized LMIS microarray platforms ideally suited for in-space micropropulsion applications ${ }^{12,13}$ (and references therein) for very small satellites or for precision control of attitude and translation maneuvers.

\section{NASA interest in liquid metal ion sources for space micropropulsion}

The most recent 2015 NASA Technology Roadmap ${ }^{14}$ identifies in-space micropropulsion technologies based on LMIS as key enabling technologies, defined as small scale systems which can deliver thrust using a conductive fluid and electrostatic fields to extract and accelerate ions, charged droplets, molecules, or clusters of molecules depending on whether the propellant is a liquid metal or ionic fluid. These systems are intended to provide high specific thrust levels characterized by low power, small form factors, and extended lifetimes. The ultimate goal is to extend current know-how gained from the fabrication and manufacture of microelectromechanical systems (MEMS) to micropropulsion platforms consisting of hundreds to thousands of needle microarrays externally wetted by a film of liquid metal, which can be electrified on demand to emit charged particles. NASA envisions that such miniaturized systems will deliver thrust levels suitable for precision attitude, rendezvous, and docking maneuvers without the use of reaction wheels. Such capability is anticipated to advance formation flight and attitude actuation for small distributed spacecraft.

The fluid dynamics responsible for cusp formation in free surface, perfectly conducting viscous liquids has therefore remained a key problem in the field of electrohydrodynamics for decades. Prior studies of cusp formation have focused almost exclusively on two asymptotic limits-the Stokes flow regime $\operatorname{Re}=0$ and the inviscid regime $\operatorname{Re}=\infty$, where $\operatorname{Re}$ denotes the Reynolds number. Since the dynamic behavior of rapidly accelerating, electrified viscous fluids represents a complex process, only these two asymptotic limits have been amenable to theoretical analysis. Such studies have shown that different forces lead to self-similar growth of the liquid tip. In this 
work, we address whether the self-similar evolution of the electrified tip persists for all Reynolds numbers Re, how do the conic apex and slope vary with $\mathrm{Re}$, and how do the exponents characterizing blowup behavior at the apex vary with $\mathrm{Re}$.

Using finite element simulations to track the shape and forces acting at the tip of an electrified protrusion in a perfectly conducting Newtonian liquid, we demonstrate that the conic tip always undergoes self-similar growth irrespective of the Reynolds number. These simulations offer detailed information about the dynamics leading to self-similar growth. The blowup exponents at the conic apex for all terms in the Navier-Stokes equation and normal stress boundary condition at the moving interface reveal the dominant forces at play as $\mathrm{Re}$ increases. Rescaling of the tip shape by the power law representing the divergence in capillary stress at the apex yields excellent collapse onto a universal cone shape with interior halfangle dependent on the Maxwell stress. The rapid acceleration of the liquid interface also generates a thin interfacial boundary layer caused by significant rate of strain. Additional details of the modeled flow, applicable to cone growth in systems such as liquid metal ion sources, help dispel prevailing misconceptions that dynamic cones resemble conventional Taylor cones or that viscous stresses at finite Re can be neglected.

\section{PREVIOUS NOTABLE STUDIES OF ELECTRIFIED CONIC FORMATION IN LIQUIDS}

\section{A. Electrostationary state known as a Taylor cone}

The early analyses of Lord Rayleigh ${ }^{16}$ and Larmor ${ }^{1}$ made evident that above a critical surface charge or critical field strength, a perfectly conducting mass of liquid will incur deformation they described as a state of "unsteady equilibrium." In 1964, Taylor posed the following question, namely, what is the equilibrium shape for which there exists a perfect balance between the capillary and Maxwell pressure everywhere on the liquid surface. He showed that this static shape is given by a cone of constant slope with an interior half-angle equal to $49.3^{\circ}$, now known as the Taylor angle. This solution, however, breaks down at the conic apex point where both pressures diverge to infinity, a feature which troubled subsequent researchers. Since then, Taylor's finding has also generated some confusion since many mistakenly believe that the dynamic cone formations observed in experimental studies, which represent rapidly advancing protrusions subject to high internal strain rates, represent Taylor cones, which by definition represent idealized static shapes. Some authors ${ }^{11}$ have attempted to resolve this confusion by referring to dynamic electrified protrusions as "Gilbert-Gray cone-jets" in honor of Gilbert, ${ }^{18}$ who first reported the attraction of water to a charged piece of amber followed by emission of a very fine jet, and Gray, ${ }^{19}$ who reported similar jet formation in water and liquid mercury as well. Unfortunately, this label has failed to stick and many researchers still refer to dynamic cones as Taylor cones.

\section{B. Zubarev analysis of self-similar conic growth in the inviscid limit $R e=\infty$}

\section{General notation}

For the remainder of this work, we note that unless otherwise stated, dimensional quantities are designated by lower case letters and dimensionless quantities by upper case letters. An exception to this is the electric field distribution to be represented in dimensional form by $\widehat{\boldsymbol{E}}$ and in dimensionless form by $\boldsymbol{E}$. Vector as well as tensor quantities are denoted by bold face variables. Subscripts denote partial differentiation. The vector $\boldsymbol{n}$ denotes a unit normal vector situated along the moving interface and pointing outward from the liquid domain.

\section{Zubarev self-similarity derivation}

In a seminal paper in 2001, Zubarev ${ }^{20}$ analyzed the dynamic distortion accompanying the response of an inviscid, perfectly conducting liquid to an initially uniform electric field. His asymptotic analysis of the unsteady Bernoulli equation describing the evolution of the velocity and electric field potential along the accelerating interface assumed incompressible irrotational flow. Key to this analysis was the assumption that the local electric field strength about the accelerating liquid tip rapidly and appreciably exceeds the applied external field strength such that local conditions prevail and the self-generated field strength decreases with distance from the apex. The external field uniformity condition was then replaced by a far field boundary condition specifying a vanishing electric field at infinity. This insight allowed Zubarev to analyze fluid motion near the apex without reference to any particular geometry, which ultimately revealed self-similar solutions for the velocity potential, electric potential, and fluid interface shape. These solutions were characterized by spatial scales that decrease in time according to $t_{\mathrm{B}}-t>0$, where $t_{\mathrm{B}}$ defined the blowup time at which the governing forces at the apex diverge to infinity. He referred to $t_{\mathrm{B}}$ as the "collapse time." We next briefly outline Zubarev's analysis leading to this prediction of self-similar blowup and corresponding blowup exponents characterizing the divergence in the Maxwell pressure, capillary pressure, and kinetic energy density at the conic apex point.

Zubarev examined the evolution of an electrified axisymmetric protrusion in a perfectly conducting inviscid fluid surrounded by vacuum where $z=h(r, z, t)$ denotes the accelerating boundary representing the vacuum/liquid interface. The electric field distribution in the vacuum domain is given by $\widehat{\boldsymbol{E}}(r, z, t)=-\nabla \phi(r, z, t)$, where the electric potential $\phi(r, z, t)$ satisfies the Laplace equation $\phi_{r r}+\phi_{r} / r+\phi_{z z}=0$ for $z \geq h(r, t)$. The velocity field in the liquid is given by $\boldsymbol{u}(r, z, t)=\nabla \psi(r, z, t)$ where the velocity potential $\psi(r, z, t)$ satisfies the equation $\psi_{r r}+\psi_{r} / r+\psi_{z z}=0$ for $z \leq h(r, t)$, as required for an incompressible liquid in irrotational flow. Given that the liquid domain is perfectly conducting, its interior domain defines a Gaussian volume devoid of electric field. The liquid interface accelerates in response to an ever increasing electrical normal force (Maxwell force), which causes fluid elongation. Conservation of mass and momentum is enforced through the unsteady form of Bernoulli's equation applied to the moving interface, which assumes inviscid and irrotational flow, given by

$$
\begin{aligned}
\rho\left(\psi_{t}\right. & \left.+\frac{\psi_{r}^{2}+\psi_{z}^{2}}{2}\right)_{z=h} \\
= & \underbrace{\epsilon_{0}\left(\frac{\phi_{r}^{2}+\phi_{z}^{2}}{2}\right)_{z=h}}_{\text {Maxwell pressure }}+\underbrace{\frac{\gamma}{\left(1+h_{r}^{2}\right)^{1 / 2}}\left(\frac{h_{r r}}{1+h_{r}^{2}}+\frac{h_{r}}{r}\right)}_{\text {Capillary pressure }},
\end{aligned}
$$


where the liquid density $\rho$ and surface tension $\gamma$ are assumed constant. The underbrace terms define the opposing local Maxwell and capillary pressures acting exclusively in the direction normal to the moving interface.

Zubarev nondimensionalized the governing equations by introducing the following scalings:

$$
\begin{gathered}
(R, Z, H)=(r, z, h) \times \frac{\epsilon_{o}}{\gamma} \widehat{E}_{\infty}^{2}, \\
T=t \times \frac{\epsilon_{o}^{3 / 2}}{\gamma \rho^{1 / 2}} \widehat{E}_{\infty}^{3}, \\
\Phi=\phi \times \frac{\epsilon_{o}}{\gamma} \widehat{E}_{\infty}, \\
\Psi=\psi \times\left(\frac{\rho \epsilon_{o}}{\gamma^{2}}\right)^{1 / 2} \widehat{E}_{\infty},
\end{gathered}
$$

where $\widehat{E}_{\infty}$ defines the asymptotic field strength such that $\phi(z$ $\rightarrow \infty)=-\widehat{E}_{\infty} z$. The boundary conditions used to solve Eq. (1) and the corresponding Laplacian fields were chosen to be

$$
\begin{array}{rl}
\Phi[R, Z, T]=0 & Z=H(R, T), \\
\lim _{\mathbf{R} \rightarrow \infty}\left(\Phi_{R}^{2}+\Phi_{Z}^{2}\right)=0 & Z \geq H(R, T), \\
\lim _{\mathbf{R} \rightarrow \infty}\left(\Psi_{R}^{2}+\Psi_{Z}^{2}\right)=0 & Z \leq H(R, T), \\
\Phi_{R}(R=0, Z, T)=0 & Z \geq H(R, T), \\
\Psi_{R}(R=0, Z, T)=0 & Z \leq H(R, T), \\
H_{R}(R=0, T)=0, & \\
H_{T}-\Psi_{Z}+\Psi_{R} H_{R}=0 & Z=H(R, T),
\end{array}
$$

where $\mathbf{R}=R \hat{\boldsymbol{e}}_{R}+Z \hat{\boldsymbol{e}}_{Z}$. Noting that the governing equations and boundary conditions are invariant to dilation by a constant $c$ for $(\Phi$, $\Psi) \rightarrow(c \Phi, c \Psi),(R, Z, H) \rightarrow\left(c^{2} R, c^{2} Z, c^{2} H\right)$, and $t \rightarrow c^{3} t$, he uncovered a set of self-similar solutions, here indicated by a tilde, according to which

$$
\begin{array}{rlrl}
{[\widetilde{R}, \widetilde{Z}, \widetilde{H}(\widetilde{R})]} & =\frac{[R, H(R, T)]}{\tau^{2 / 3},} \\
\widetilde{\Phi}(\widetilde{R}, \widetilde{Z}) & =\frac{\Phi(R, Z, T)}{\tau^{1 / 3}} \quad \widetilde{Z} \geq \widetilde{H}(\widetilde{R}), \\
\widetilde{\Psi}(\widetilde{R}, \widetilde{Z}) & =\frac{\Psi(R, Z, T)}{\tau^{1 / 3}} & \widetilde{Z} \leq \widetilde{H}(\widetilde{R}), \\
\text { where } \tau & =T_{\mathrm{B}}-T
\end{array}
$$

and $T_{\mathrm{B}}$ denotes the dimensionless blowup time.

Zubarev constructed self-consistent solutions to these equations by matching the self-similar solutions in the inner region of the liquid apex to the far field solutions describing the conventional static Taylor cone with interior half-angle $\theta_{o}=49.3^{\circ}$. To leading order $\bar{R}=\left(\widetilde{R}^{2}+\widetilde{Z}^{2}\right)^{1 / 2} \rightarrow \infty$ (or equivalently $\tau \rightarrow 0$ in selfsimilar coordinates), he found that the asymptotic functions (more conveniently solved using spherical coordinates) scaled as

$$
\widetilde{\Psi}(\widetilde{R}, \widetilde{Z}) \propto s / \bar{R}
$$

$$
\begin{gathered}
\widetilde{\Phi}(\widetilde{R}, \widetilde{Z}) \propto \frac{1}{c_{o}}\left[2 \bar{R}\left(s_{o}-s\right)\right]^{1 / 2} P_{1 / 2}(\cos \theta), \\
\widetilde{H}(\widetilde{R})=-s_{o} \widetilde{R},
\end{gathered}
$$

where

$$
\begin{gathered}
P_{1 / 2}\left(\cos \theta_{o}\right)=0, \\
s_{o}=-\cot \theta_{o}, \\
c_{o}=\left(\frac{d P_{1 / 2}(\cos \theta)}{d \theta}\right)_{\theta=\theta_{o}},
\end{gathered}
$$

where $\theta=\arctan (\widetilde{R} / \widetilde{Z})$ denotes the polar angle in spherical coordinates, $P_{1 / 2}(\cos \theta)$ denotes the Legendre polynomial of order $1 / 2$, and $s$ is a constant such that $0<s<s_{0}$. As evident from Eq. (17), the solution for the velocity potential corresponds to fluid motion that is spherically symmetric such that liquid is transported to the conic apex by streamlines that are always tangent to the moving interface. Zubarev noted that the apex point $(\widetilde{R}, \widetilde{Z})=(0,0)$ therefore acts as a point sink for the flow.

These self-similar solutions describe the divergent behavior of the Maxwell pressure $P_{M}$, capillary pressure $P_{C}$, and flow speed $|\boldsymbol{U}|$ at the advancing conic apex, which therefore scale according to

$$
\begin{aligned}
P_{M} \sim P_{C} & \sim \tau^{-2 / 3}, \\
|\boldsymbol{U}| & \sim \tau^{-1 / 3} .
\end{aligned}
$$

Zubarev thereby demonstrated for the first time that the conic apex region of a perfectly conducting liquid protrusion, subject to inviscid, irrotational, and incompressible flow and under the sole action of Maxwell and capillary forces, will undergo self-similar growth. He described the evolving shape as a dynamic conic cusp.

\section{Simulations of self-similar conic growth in viscous liquids}

\section{Numerical studies by Suvorov, Litvinov, and Zubarev}

Around the time Zubarev reported his findings for inviscid flow, Suvorov and Litvinov ${ }^{21}$ published a computational study aimed at understanding the influence of viscous forces using the axisymmetric model shown in Fig. 2. They employed a Marker and Cell (MAC) technique coupled with a coordinate transformation for adaptive grid generation, ${ }^{22}$ which allowed smooth and stable evolution of the vacuum/liquid interface even in the region of the conic apex subject to significant and rapidly increasing curvature. This technique successfully suppressed spurious surface oscillations in regions of high curvature, which had plagued earlier researchers. The MAC method had also been previously used by Zheng and Linsu $^{23}$ who reported that perfectly conducting viscous electrified liquids tend to distort into conelike shapes with interior half-angles not equal to the Taylor cone angle, though the descriptions provided were brief and lacked detailed results.

As shown in Fig. 2, Suvorov and Litvinov ${ }^{21}$ partitioned the axisymmetric volume of interest into two contacting domains-an upper domain comprising the vacuum region subject to an initial vertical uniform electric field of strength $E_{o}=2.4 \times 10^{8} \mathrm{~V} / \mathrm{m}$ and 
a lower domain comprising a perfectly conducting, incompressible Newtonian liquid. Given the large disparity in density and viscosity, the hydrodynamics of the vacuum domain was neglected. The normal component of the electric field strength along the vacuumliquid interface (held at constant electric potential) was updated in time according to its spatiotemporal coordinates. The Navier-Stokes equation for the liquid domain was solved self-consistently subject to the kinematic boundary condition governing the motion of the moving interface. The initial thickness and radius of the vacuum and liquid domain were set to $20 \mu \mathrm{m}$. The material constants of the liquid were chosen to match those of liquid mercury. The initial condition for the vacuum/liquid interface was chosen to be a flat film with a small Gaussian bump centered at the origin given by $h(r, t=0)=a$ $\exp \left[-\left(r^{2} / \lambda^{2}\right)\right]$, where $a=0.4$ or $4.0 \mu \mathrm{m}$ and $\lambda=4 \mu \mathrm{m}$. The authors indicated that this choice of dimensions, material constants, and operating parameters yielded a value $\mathrm{Re}=717$ describing laminar flow.

For an initial Gaussian bump with a fixed lateral extent, simulations showed that a thicker liquid layer tended to produce a more rounded protrusion that advanced rapidly in time. By contrast, a thinner liquid layer yielded a more pronounced cuspidal shape that advanced more slowly, presumably due to stronger viscous effects. The cuspidal shapes manifest a conic tip whose apical radius of curvature was observed to shrink rapidly in time. Suvorov and Litvinov called these structures dynamic Taylor cones to emphasize that such shapes do not resemble conventional stationary Taylor cones, as highlighted previously by Zheng and Linsu ${ }^{23}$ who called the evolving dynamic shapes "transitional states." Suvorov and Litvinov also carried out simulations in which the viscous term in the normal stress boundary condition was altogether omitted. They concluded from those studies that the viscous normal force at the apex is always positive-that is, it too acts in the same direction as the capillary force though it always appeared to be much smaller in magnitude. They reported that the resulting shapes obtained with or without the viscous normal stress were similar, but no comparative images were provided.

The numerical scheme upon which this work was based, which allowed stable interface growth over half a decade in time, successfully captures divergent behavior at the conic apex of such quantities as the curvature radius, electric field strength, and axial velocity. These divergent quantities evidenced runaway behavior in the Maxwell pressure, capillary pressure, and kinetic energy density. This study demonstrated that the (negative) Maxwell normal stress pulling upward on the liquid tip rapidly overtakes the (positive) normal stresses due to capillary and viscous forces for the chosen parameter set. Suvorov and Litvanov urged that more computational studies be conducted to assist theorists developing analytic treatments of electrohydrodynamic behavior for LMIS applications. While their work established key features characterizing runaway cusp formation in viscous fluids, their study was restricted to a single value of Re. They also did not report the actual blowup exponents found for the divergent forces acting at the accelerating apex.

Soon thereafter, there followed similar computational studies by Suvorov ${ }^{24}$ and Suvorov and Zubarev ${ }^{25}$ simulating the behavior of liquid gallium at the melting point at flow conditions corresponding to even smaller Reynolds number. In these studies, the viscous normal stress term in the interface boundary condition was altogether omitted based on earlier indications ${ }^{21}$ that the viscous term in the normal stress boundary condition tended to be much smaller than the capillary term. In these simulations, the initial thickness and radius of the vacuum and liquid domain were reduced to $5 \mu \mathrm{m}$ and the initial applied electric field strength doubled in value to $E_{o}=4.8$ $\times 10^{8} \mathrm{~V} / \mathrm{m}$. The initial condition for the interface was again chosen to be a flat film with a small Gaussian bump centered at the origin of the form $h(r, t=0)=a \exp \left[-\left(r^{2} / \lambda^{2}\right)\right]$ where $a=0.1 \mu \mathrm{m}$ and $\lambda=1 \mu \mathrm{m}$. These Gaussian parameters, corresponding to an initial radius of curvature of the liquid protrusion equal to $\lambda^{2} / 4 a=2.5 \mu \mathrm{m}$, were chosen to approximate the radii of needles in typical liquid metal ion sources. The chosen dimensions and material constants in these studies corresponded to a value $\operatorname{Re}=187$.

The numerical results by Suvorov ${ }^{24}$ are shown in Fig. 1. Only this set of figures is discussed here since the results in Ref. 25 are similar. Snapshots of the interface shape in Figs. 1(a) and 1(b) illustrate both far field and near field views of an accelerating protrusion. These images reveal how a rapidly accelerating and sharpening interface undergoes self-similar collapse toward a conic tip with a rapidly decreasing radius of curvature. The curves in Fig. 1(c) illustrate blowup behavior in the magnitude of the Maxwell and the capillary pressure at the conic apex as the system approaches the blowup time of 123.542 ns. Figure 1(d) represents a double logarithmic plot of the magnitude of the Maxwell pressure, capillary pressure, and kinetic energy density at the conic apex upon approach to blowup. The solid lines shown were reported to yield exponent values for the Maxwell pressure, capillary pressure, and kinetic energy density of $0.67,0.68$, and 0.63 , respectively. The dashed lines we have superposed represent exponent values $0.70,0.77$, and 0.78 , respectively, which appear to provide a better fit to the data.

\section{More recent numerical studies of conic tip growth in electrified liquids}

More recently, Collins et al. ${ }^{26,27}$ have used finite element simulations with elliptic mesh generation and adaptive time integration to simulate liquid cone growth in perfectly conducting, perfectly insulating, and leaky dielectric liquids. They conducted these simulations to understand why electrohydrodynamic tip streaming resulting in the emission of a thin fluid jet from the conic tip does not occur in perfectly conducting or perfectly insulating liquids. Simulations of perfectly conducting liquids did reveal selfenhanced tip sharpening (with no tip streaming) with divergent behavior in the Maxwell, capillary, and viscous normal stress at the conic apex, as reported previously; $;^{21,24,25}$ unfortunately, the values of the blowup exponents were not reported. Interestingly, their studies confirmed that tip-streaming requires tangential Maxwell surface stresses near the conic tip. (Holgate and Coppins ${ }^{28}$ have also recently confirmed that any tip streaming observed in numerical simulations of viscous, perfectly conducting liquids is caused by spurious numerical effects since perfectly conducting liquids cannot support tangential Maxwell stresses along the interface. They demonstrated this by correlating droplet size from tip streaming with the local grid size-see authors' comment in Fig. 5.) The simulations of Collins et al. involving leaky dielectric liquids, which support tangential surface stresses, also confirmed the scaling laws first predicted by de la Mora and Loscertales and Gañán-Calvo $^{29,30}$ for the size of droplets emanating from steady cone-jets. 

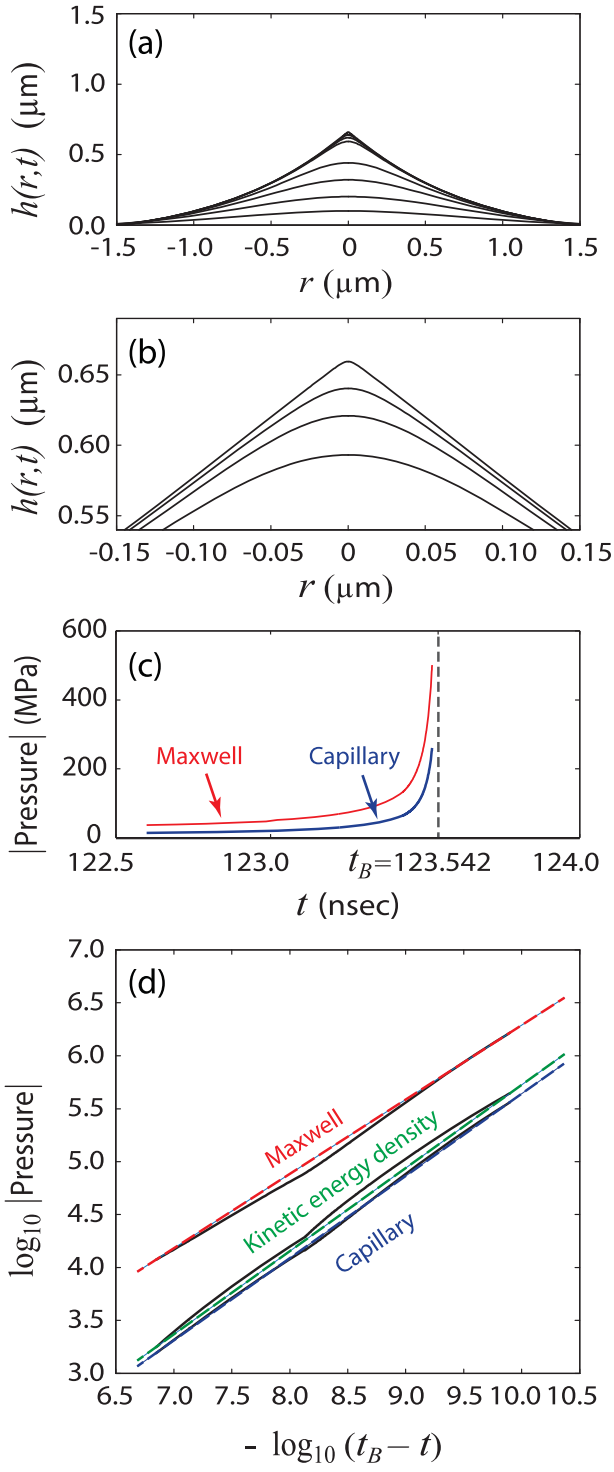

FIG. 1. Self-similar growth of an electrified liquid cusp in a thin film of gallium ( $5 \mu \mathrm{m}$ in thickness) with an initial Gaussian bump of amplitude $0.1 \mu \mathrm{m}$ centered about the origin and subject to an initial uniform vertical electric field of strength $E_{0}=4.8 \times 10^{8} \mathrm{~V} / \mathrm{m}$. Reproduced with permission from V. G. Suvorov, "Numerical analysis of liquid metal flow in the presence of an electric field: Application to liquid metal ion source," Surf. Interface Anal. 36, 423 (2004). Copyright 2004 John Wiley and Sons. (a) Interface shapes at times $t=0,81.2,104.5,116.1,122.6$, $123.1,123.4$, and $123.5 \mathrm{~ns}$. (b) Magnified view of the conic tip at $t=122.6,123.1$, 123.4 , and $123.5 \mathrm{~ns}$. (c) Divergent behavior of the (negative) Maxwell pressure and (positive) capillary pressure at the conic apex upon approach to the blowup time $t_{\mathrm{B}}=123.542 \mathrm{~ns}$. (d) Log-log plot showing power law growth of the (dimensionless) Maxwell pressure, capillary pressure, and kinetic energy density as $t_{\mathrm{B}}$ is approached.

Burton and Taborek ${ }^{31}$ also conducted detailed boundary integral simulations to examine Coulombic fission of charged inviscid droplets. Their studies revealed how small disturbances cause rapid elongation of an initial slightly prolate ellipsoidal droplet to produce polar cap regions resembling conic tips, which they coined lemonshaped drops. For perfectly conducting liquids, they showed how conic tips undergo self-similar sharpening due to local field selfenhancement with a corresponding blowup exponent of $2 / 3$ for the capillary pressure and $1 / 3$ for the surface charge density. Remarkably, they were able to confirm self-similarity over 12 decades in time-an extraordinarily long period-requiring stable integration in order to resolve the very fine scales at the advancing tip. Incidentally, they too reported that during conic sharpening, the interior half-angle never equals the Taylor cone static angle of $49.3^{\circ}$, except as they suggested at the very moment of blowup.

Different interface tracking schemes have since been implemented by other groups interested in similar systems. For example, Garzon, Gray, and Sethian ${ }^{32}$ have examined the behavior of perfectly conducting, inviscid droplets in an initial uniform external electric field. They implemented boundary integral simulations to solve for the flow velocity, Maxwell pressure, and capillary pressure along the free surface and a level set method for the Eulerian potential flow model for tracking droplet evolution past breakup. Depending on the value of the initial field strength, the resulting exponents at the apical tip for the Maxwell pressure, capillary pressure, and kinetic energy density were found to range from 0.662 to $0.676,0.57$ to 0.62 (smaller precision reported due to larger fluctuations), and 0.672 to 0.736 , respectively. In all cases examined, larger initial field strengths produced conic tips with larger interior halfangles, which were always smaller than the Taylor cone static angle of $49.3^{\circ}$.

\section{Self-similar conic tip growth in the Stokes regime $(\mathrm{Re}=0)$}

Additional studies during the past decade have examined the role of viscous forces in the formation and growth of conic cusps. Fontelos and co-workers ${ }^{33,34}$ employed boundary integral techniques for tracking the distortion of perfectly conducting droplets subject to viscous effects. By focusing exclusively on the Stokes regime $R e=0$, they showed that for a sufficiently large surface charge and/or sufficiently large external field, the polar caps of an initially rounded droplet will deform into conic tips through a self-similar process. For the parameter set chosen in these studies, the interior half-angles of conic tips ranged from about $25^{\circ}$ to $30^{\circ}$, much smaller than the Taylor angle static value of $49.3^{\circ}$, irrespective of the values of the actual surface charge or field strength. These studies indicate that viscous effects tend to repress fluid tips from undergoing excessive sharpening.

Fontelos and co-workers ${ }^{33}$ also examined numerically the deformation of an isolated charged droplet of a perfectly conducting viscous liquid embedded within a dielectric viscous medium. For cases in which all the electric charge resides at the liquid/liquid interface, the surface charge density is given by $\sigma=-\epsilon_{o} \partial V / \partial n$, where $V$ is the electric potential and $\partial / \partial n$ denotes the normal derivative along the free surface. The repulsive electrical force per unit area is then given by $\boldsymbol{F}=\sigma \widehat{\boldsymbol{E}} / 2=\left(\epsilon_{o} / 2\right)(\partial V / \partial n)^{2} \boldsymbol{n}=\left(\sigma^{2} / 2 \epsilon_{o}\right) \boldsymbol{n}$. The interior $(i=1)$ and exterior $(i=2)$ liquid domains were made to satisfy the Stokes equation and continuity equations given by

$$
\begin{gathered}
\mu_{i} \nabla^{2} \boldsymbol{u}_{i}-\nabla p_{i}=0, \\
\nabla \cdot \boldsymbol{u}_{i}=0 .
\end{gathered}
$$


The velocity and pressure fields along the free surface were made to satisfy the normal stress boundary condition

$$
p_{1}-p_{2}=\gamma \nabla \cdot \boldsymbol{n}-\frac{\sigma^{2}}{2 \epsilon_{o}}+\boldsymbol{n} \cdot\left(\boldsymbol{\tau}_{1}-\boldsymbol{\tau}_{2}\right) \cdot \boldsymbol{n},
$$

as well as the tangential stress boundary condition

$$
\boldsymbol{t} \cdot\left(\tau_{2}-\tau_{1}\right) \cdot \boldsymbol{n}=0,
$$

where $\gamma$ denotes the interfacial tension of the liquid/liquid interface, $\boldsymbol{\tau}_{i}$ is the deviatoric stress tensor for a Newtonian liquid given by

$$
\boldsymbol{\tau}_{i}=\mu_{i}\left[\nabla \boldsymbol{u}_{i}+\left(\nabla \boldsymbol{u}_{i}\right)^{T}\right]
$$

$T$ denotes the matrix transpose, $\boldsymbol{n}$ is the local unit surface normal vector pointing away from medium 1 to 2 , and $\boldsymbol{t}$ denotes each of two orthogonal unit surface tangent vectors. The capillary stress in Eq. (27) can also be rewritten in terms of the mean curvature of the deformed interface $\mathcal{K}$ given by ${ }^{35}$

$$
\mathcal{K}=-\frac{1}{2} \nabla \cdot \boldsymbol{n}
$$

Their numerical results and supporting scaling arguments ${ }^{33}$ showed that for isolated charged droplets, the interface curvature, charge density, electric field, and velocity at the conic apex diverge according to $\tau^{-1 / 2}$ as $\tau=t_{B}-t \rightarrow 0$.

In a follow-up study, ${ }^{34}$ they examined an isolated charged droplet in an external uniform electric field and showed that for self-similarity to persist toward blowup, the surface change density must scale as $\tau^{-1 / 2}$, the tip curvature must scale as $\tau^{-\alpha}$, and the tip velocity must scale as $\tau^{\alpha-1}$, where $\alpha>0$ represents a given function dependent on the far field value of the cone interior half-angle. Their studies revealed that the external field acting on a charged droplet represents only a vanishingly small perturbation to the dominant divergent field at the apical tip caused by the accumulation of local surface charge in that region. It was for this reason that the interior half-angle of charged droplets was found to be essentially independent of the value of the external field.

More importantly, Fontelos and co-workers emphasized that in the limit of vanishingly small $\mathrm{Re}$, the dominant force balance responsible for self-similar evolution of the conic tip is not due to opposing Maxwell and capillary stresses but opposing Maxwell and viscous stresses. From the leading order balance between viscous and Maxwell forces in Eq. (27), they concluded that the tip electric field strength must scale as $\tau^{-1 / 2}$, which in turn requires that the tip curvature scale as $\tau^{-\alpha}$ and the tip velocity scale as $\tau^{\alpha-1}$, where the self-similar coordinate is given by $\boldsymbol{\xi}=\boldsymbol{r} / \tau^{\alpha}$.

In a separate calculation, they solved for the velocity and electric field distributions within an infinite cone of perfectly conducting fluid. They obtained two solutions for the velocity field-one describing symmetric formation of cusps at the poles of an isolated charged droplet or an uncharged droplet in an external electric field and the other describing asymmetric formation of a single cusp. In both cases, the velocity field, which was found to scale as $\xi^{(\alpha-1) / \alpha}$ (derivation not shown), was required to smoothly and asymptotically match the far field velocity function for a liquid cone at equipotential subject to vanishing surface shear stress and vanishing normal stress, which scales as $r^{\lambda}$, yielding the relation $(\alpha-1) / \alpha=\lambda$. In the symmetric case, the scaling parameter $\lambda=-1$, which yielded the value $\alpha=1 / 2$. In the asymmetric case, the value $\lambda$ was found to depend on the interior cone angle value such that neither $\lambda$ nor $\alpha$ could be expressed as simple integer ratios. Their simulations produced relatively constant values of the cone interior half-angle of about $27.5^{\circ}$ for a wide range of external fields, corresponding roughly to a value $\alpha=-0.74$. This value of $\alpha$ corresponded closely with the exponent for the apex curvature extracted from their simulations.

\section{COMPUTATIONAL MODEL FOR CONIC CUSP GROWTH IN ELECTRIFIED VISCOUS LIQUID LAYER}

As discussed above, both the inviscid $\operatorname{Re}=\infty$ limit and the Stokes $\mathrm{Re}=0$ limit permit analytic treatment, resulting in exponents characterizing divergent self-similar growth of the conic apex in perfectly conducting droplets and films. These calculations have shown that while the dominant opposing normal stresses at $\mathrm{Re} \rightarrow \infty$ are the Maxwell pressure and capillary pressure, the dominant opposing stresses at $\operatorname{Re}=0$ transition to a competition between the Maxwell pressure and the viscous normal stress. In the work which follows, we demonstrate that self-similar evolution of the electrified tip persists for all Reynolds numbers despite the transition in dominant competitive forces. From our simulations, we extract the corresponding blowup exponents characterizing the various forces acting at the conic apex and demonstrate how those exponents vary with increasing Reynolds number Re (at a fixed capillary number $\mathrm{Ca}$ ). The computed interface shapes also reveal how the interior cone angle upon approach to blowup varies with $\operatorname{Re}$ (at a fixed capillary number $\mathrm{Ca}$ ).

Shown in Fig. 2 is a sketch of the axisymmetric geometry used in our study. The system consists of a perfectly conducting layer of liquid of radius and initial thickness $h_{o}$ held at constant potential

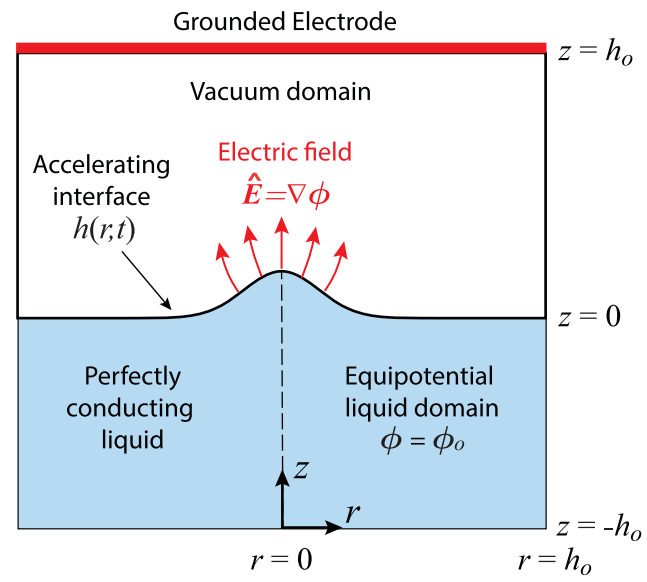

FIG. 2. Geometry for computational study. Circular layer of perfectly conducting liquid with radius and thickness equal to $h_{0}$ held at constant potential $\phi_{0}$. Initial separation distance between the liquid layer and the grounded circular counter electrode is $h_{0}$. Initial liquid configuration chosen to be a flat layer with a small Gaussian bump centered about the origin. Protrusion rapidly accelerates toward the counter electrode due to self-enhancement of the electric field at the liquid apex. 
$\phi_{o}$. The initial liquid layer is separated from a grounded cylindrical electrode of radius $h_{o}$ by a vacuum layer of thickness $h_{o}$. This is the same geometry used in the original study of Suvorov and Litvinov ${ }^{21}$ and Suvorov and Zubarev.

Since charge transport in perfectly conducting liquids occurs instantaneously by comparison to the time scale for viscous flow, the electrostatic approximation $\nabla \cdot \widehat{\boldsymbol{E}}=0$ is valid in the vacuum domain $\left(0 \leq r \leq h_{o}, h(r, t) \leq z \leq h_{o}\right)$, where the electric potential $\phi(r, z, t)$ satisfies the equation

$$
\phi_{r r}+\frac{\phi_{r}}{r}+\phi_{z z}=0
$$

subject to the boundary conditions

$$
\begin{gathered}
\phi\left[0 \leq r \leq h_{o}, z=h(r, t)\right]=\phi_{o}, \\
\phi\left(0 \leq r \leq h_{o}, z=h_{o}, t\right)=0, \\
\frac{\partial \phi}{\partial r}\left[r=0, h(r, t) \leq z \leq h_{o}, t\right]=0, \\
\frac{\partial \phi}{\partial r}\left[r=h_{o}, h(r, t) \leq z \leq h_{o}, t\right]=0 .
\end{gathered}
$$

Accordingly, the electric field increases in time in response to the advance of the equipotential boundary $h(r, z, t)$ toward the grounded electrode. Any protrusion along the liquid interface therefore undergoes local accelerated growth due to field enhancement at the tip.

The hydrodynamic behavior of the electrified liquid is described by the Navier-Stokes equation for an incompressible, Newtonian liquid where the velocity field $\boldsymbol{u}(r, z, t)=u(r, z, t) \hat{\boldsymbol{e}}_{r}$ $+v(r, z, t) \hat{\boldsymbol{e}}_{z}$ and pressure field $p(r, z, t)$ satisfy the governing mass and momentum conservation equations given by

$$
\begin{aligned}
\nabla \cdot \boldsymbol{u} & =0, \\
\rho\left(\frac{\partial \boldsymbol{u}}{\partial t}+(\boldsymbol{u} \cdot \nabla) \boldsymbol{u}\right) & =-\nabla p+\mu \nabla^{2} \boldsymbol{u} .
\end{aligned}
$$

For simplicity, gravitational effects can be neglected so long as the Bond number Bo $=\rho g h_{o}^{2} / \gamma<<1$. Within the liquid domain $[0 \leq r$ $\left.\leq h_{o},-h_{o} \leq z \leq h(r, t)\right]$, the velocity components $u(r, z, t)$ and $v(r, z, t)$ must satisfy the following boundary conditions prescribed along the central axis $r=0$, bottom $\left(z=-h_{o}\right)$, and side wall $\left(r=h_{o}\right)$ :

$$
\begin{array}{rr}
\left.u\right|_{r=0}=0, & \text { symmetry, } \\
\left.\frac{\partial v}{\partial r}\right|_{r=0}=0, & \text { symmetry, } \\
\left.u\right|_{r=h_{o}}=0, & \text { inpenetrability, } \\
\left.v\right|_{z=-h_{o}}=0, & \text { inpenetrability, } \\
\left.u\right|_{z=-h_{o}}=0, & \text { no slip, } \\
\left.v\right|_{r=h_{o}}=0, & \text { no slip. }
\end{array}
$$

The two remaining boundary conditions for $(u, v)$ specify vanishing shear stress along the free surface $z=h(r, z, t)$, namely,

$$
[\boldsymbol{n} \cdot \boldsymbol{\tau} \cdot \boldsymbol{t}]_{z=h(r, t)}=0
$$

where $\boldsymbol{\tau}$ is the deviatoric stress tensor defined in Eq. (29). The boundary condition for the pressure field along the free surface is enforced by the interface normal stress boundary condition

$$
\left.p\right|_{z=h(r, t)}=\left[-2 \gamma \mathcal{K}-\frac{\epsilon_{o}}{2} \widehat{E}_{n}^{2}+\boldsymbol{n} \cdot \boldsymbol{\tau} \cdot \boldsymbol{n}\right]_{z=h(r, t)},
$$

where $\widehat{E}_{n}=\widehat{\boldsymbol{E}} \cdot \boldsymbol{n}$ and the local mean curvature of the free surface defined by Eq. (30) is given by

$$
\begin{gathered}
\mathcal{K}=\frac{1}{2 b^{3}}\left(\frac{d^{2} h(r, t)}{d r^{2}}+\frac{b^{2}}{r} \frac{d h}{d r}\right), \\
b=\left[1+\left(\frac{d h(r, t)}{d r}\right)^{2}\right]^{1 / 2} .
\end{gathered}
$$

The terms on the right hand side of Eq. (45) represent the capillary pressure, Maxwell pressure, and viscous normal stress, respectively. The sign convention is such that the capillary pressure at the apex is always positive and repressive in nature due to the local concave shape, while the Maxwell pressure is always negative and continually pulls the apex toward the counter electrode. The position of the advancing interface is obtained from the kinetic boundary condition that requires

$$
\left.v\right|_{z=h(r, t)}=\frac{\partial h}{\partial t}+\left.\boldsymbol{u}\right|_{z=h(r, t)} \cdot \nabla_{s} h(r, t),
$$

where $\nabla_{s}=(\mathbb{I}-\boldsymbol{n} \boldsymbol{n}) \cdot \nabla$ denotes the surface gradient operator, $\mathbb{I}$ is the identity tensor, and

$$
\left.\boldsymbol{u}\right|_{z=h(r, t)} \cdot \nabla_{s} h=\left[\frac{u h_{r}}{1+h_{r}^{2}} \hat{\boldsymbol{e}}_{r}+\frac{v h_{r}^{2}}{1+h_{r}^{2}} \hat{\boldsymbol{e}}_{z}\right]_{z=h(r, t)} .
$$

The initial condition for the velocity field represents a quiescent state $\boldsymbol{u}(r, z, t)=0$. The initial surface configuration is chosen to be a flat liquid layer with a small Gaussian bump at the origin,

$$
h\left(0 \leq r \leq h_{o}, t=0\right)=h_{o}+a_{o} e^{-(r / \lambda)^{2}} .
$$

The peak Gaussian amplitude and lateral extent scale uniformly with $h_{o}$ such that $a_{o}=0.02 h_{o}$ and $\lambda=0.2 h_{o}$.

\section{A. Scaling and nondimensionalization}

To probe the influence of inertial and viscous effects on the process of conic cusp formation, we implemented the scalings in Table I to convert equations and boundary conditions to dimensionless form. In addition to the parameter values specified in Fig. 2, the material constants characterizing the liquid metal, namely, $\rho$, $\mu$, and $\gamma$, represent the liquid density, viscosity, and surface tension, respectively. The dimensionless Navier-Stokes equation is then given by

$$
\frac{\partial \boldsymbol{U}}{\partial T}+\boldsymbol{U} \cdot \widetilde{\nabla} \boldsymbol{U}=-\widetilde{\nabla} P+\frac{1}{\operatorname{Re}} \widetilde{\nabla}^{2} \boldsymbol{U}
$$

subject to the (dimensionless) normal stress boundary condition at the moving interface,

$$
\left.P\right|_{Z=H(R, T)}=\frac{1}{\mathrm{Ca}}(\widetilde{\nabla} \cdot \widetilde{\boldsymbol{n}})-E_{\widetilde{n}}^{2}+\frac{1}{\operatorname{Re}} \widetilde{\boldsymbol{n}} \cdot \widetilde{\boldsymbol{\tau}} \cdot \widetilde{\boldsymbol{n}},
$$


TABLE I. Characteristic (c) scalings (lower case symbols) and nondimensional variables (uppercase symbols) for model geometry depicted in Fig. 2.

\begin{tabular}{|c|c|c|}
\hline Quantity & Scaling & $\begin{array}{l}\text { Rescaled } \\
\text { variable }\end{array}$ \\
\hline Coordinates & $\begin{array}{l}r_{c}=h_{o} \\
z_{c}=h_{o}\end{array}$ & $\begin{array}{l}R=r / r_{c} \\
Z=z / z_{c}\end{array}$ \\
\hline Interface shape & & $H=h / z_{c}$ \\
\hline $\begin{array}{l}\text { Apex height }\left(T=T_{f}\right) \\
\text { Apex height }\left(T=T_{\mathrm{B}}\right)\end{array}$ & & $\begin{array}{l}Z_{f}=z_{f} / z_{c} \\
Z_{\mathrm{B}}=z_{\mathrm{B}} / z_{c}\end{array}$ \\
\hline Operators & & $\begin{aligned} \widetilde{\nabla} & =h_{o} \nabla \\
\widetilde{\nabla}^{2} & =h_{o}^{2} \nabla^{2}\end{aligned}$ \\
\hline Electric potential & $\phi_{c}=\phi_{o}$ & $\Phi=\phi / \phi_{c}$ \\
\hline Electric field strength & $\begin{aligned} E_{c} & =\phi_{c} / z_{c} \\
& =\phi_{o} / h_{o}\end{aligned}$ & $\boldsymbol{E}=\widehat{\boldsymbol{E}} / E_{c}$ \\
\hline Fluid pressure & $\begin{aligned} p_{c} & =\epsilon_{o} E_{c}^{2} / 2 \\
& =\epsilon_{o} \phi_{o}^{2} /\left(2 h_{o}^{2}\right)\end{aligned}$ & $P=p / p_{c}$ \\
\hline \multirow[t]{2}{*}{ Fluid velocity } & $\begin{aligned} u_{c} & =\sqrt{p_{c} / \rho} \\
& =\sqrt{\epsilon_{o} / 2 \rho}\left(\phi_{o} / h_{o}\right)\end{aligned}$ & $U=u / u_{c}$ \\
\hline & $v_{c}=u_{c}$ & $V=v / v_{c}$ \\
\hline Time & $\begin{aligned} t_{c} & =h_{o} / v_{o} \\
& =\sqrt{2 \rho / \epsilon_{o}}\left(h_{o}^{2} / \phi_{o}\right)\end{aligned}$ & $T=t / t_{c}$ \\
\hline Final simulation time & & $T_{f}=t_{f} / t_{c}$ \\
\hline Asymptotic blowup time & & $T_{\mathrm{B}}=t_{B} / t_{c}$ \\
\hline $\begin{array}{l}\text { Time interval for } \\
\text { self-similar scaling }\end{array}$ & & $\tau=T_{\mathrm{B}}-T$ \\
\hline Reynolds number & $\begin{aligned} \operatorname{Re} & =\rho v_{c} z_{c} / \mu \\
& =\sqrt{\epsilon_{o} \rho / 2 \mu^{2}} \phi_{o}\end{aligned}$ & \\
\hline Capillary number & $\begin{aligned} \mathrm{Ca} & =p_{c} z_{c} / \gamma \\
& =\left(\epsilon_{o} / 2 \gamma\right)\left(\phi_{o}^{2} / h_{o}\right)\end{aligned}$ & \\
\hline Bond number & $\mathrm{Bo}=\rho g h_{o}^{2} / \gamma$ & \\
\hline
\end{tabular}

where $E_{\widetilde{n}}=\boldsymbol{E} \cdot \widetilde{\boldsymbol{n}}$ and $\widetilde{\boldsymbol{n}}=\left(-H_{R} \hat{\boldsymbol{e}}_{R}+\hat{\boldsymbol{e}}_{Z}\right) / \sqrt{1+H_{R}^{2}}$. In rescaled form, the initial interface shape is given by

$$
H(R, T=0)=A_{o} e^{-R^{2} / \Lambda^{2}},
$$

where the peak amplitude and extent are denoted by $A_{o}=0.02$ and $\Lambda=0.2$. The remaining dimensionless equations and boundary conditions are easily obtained and not restated here.

\section{B. Influence of inertial and viscous effects for increasing $\mathrm{Re}$ at fixed $\mathrm{Ca}$}

From Eqs. (51) and (52), it is evident that both the Reynolds and capillary numbers play a key role in the growth process. For too small values of the capillary number, the flow will be overwhelmed by the repressive influence of surface tension such that budding protrusions are rapidly leveled, thereby suppressing formation of fluid elongations. By contrast, too large values of $\mathrm{Ca}$ cause formation of ripples along the moving interface, which complicate analysis due to interference effects between multiple growing protrusions. In our study, we therefore chose to fix the capillary number at an intermediate value which allowed formation and stable growth of a liquid protrusion. To that end, we chose the same value used by Suvorov and Zubarev, ${ }^{24,25}$ namely, $\mathrm{Ca}=7.0834$. We extracted this value from their studies by appealing to the definition of $\mathrm{Ca}$ in Table I and noting the values $\gamma=0.72 \mathrm{~N} / \mathrm{m}, h_{o}=5 \mu \mathrm{m}$, and $\phi_{o}=2.4 \mathrm{kV}$ used in those studies.

\section{Consequences of holding Ca constant in simulations}

In the results that follow, it is important to understand the consequences of holding $\mathrm{Ca}$ fixed when examining the flow behavior and liquid shapes obtained at increasing values of Re. In particular, given a liquid with specified material constants, the restriction of fixed capillary number imposes the constraint that the ratio $\phi_{o}^{2} / h_{o}$ remains constant. Therefore, an increase in $\mathrm{Re}$ by a factor $f$ reflects an increase in the voltage potential to $f \phi_{o}$ and an increase in the characteristic system size $h_{o}$ to $f^{2} h_{o}$. According to the scalings in Table I, these increases lead to an effective decrease in the initial field strength from $E_{o}=\phi_{o} / h_{o}$ to $E_{o} / f$ and a corresponding decrease in the initial flow speed by a factor $1 / f$.

\section{Details of numerical simulations}

To solve the coupled equations and boundary conditions discussed in Sec. III, we employed finite element simulations using the commercial software package $\mathrm{COMSOL}^{36}$ using its arbitrary Lagrangian-Eulerian method for coupling the vacuum and liquid domains across the moving interface. The computational model was constructed assuming incompressible laminar flow within the liquid domain, modeled as a perfect liquid conductor in contact with an inert vacuum domain. The liquid domain therefore contained no internal electric field, and the electric field at the vacuum/liquid interface was always everywhere oriented in the direction normal and away from the moving interface. The electric potential distribution within the vacuum domain and on the liquid surface was updated at each time step according to the local position of the moving boundary modeled by a moving mesh. At each time step, all mesh element edges along the vacuum/liquid boundary were translated at a distance given by the local speed of the liquid. All interior mesh elements were reconfigured by a uniform Laplace smoothing process to ensure that boundary displacement and subsequent fluid motion proceeded smoothly throughout the liquid domain. The entire mesh was reconstituted whenever the mesh quality of the most distorted element fell below a specified value. Mesh generation relied on quadratic triangular Lagrangian elements for the velocity field and linear elements for the pressure field, so-called $P 2+P 1$ mixed order discretization. Second-order elements provided higher resolution with stability even at the highest Reynolds number studied.

Two different nonuniform meshes were employed, one optimized for low $\operatorname{Re}$ (mesh A) and the other optimized for high $\operatorname{Re}$ (mesh B) behavior. Flow at intermediate values of Re was found to yield the same results with either meshing scheme. For mesh $A$, the edge length of mesh elements adjacent to the moving interface was set equal to the value $0.004 r_{o}$, where $r_{o}$ denotes the local 
interface radius of curvature, subject to an upper and a lower bound. Additionally, at very late times where the curvature of the advancing cone increased rapidly, the mesh in the vicinity of the conic tip was iteratively refined by further subdividing the triangular elements to achieve satisfactory resolution. To enforce mesh refinement, the final position of the conic tip was first estimated from simulations conducted with a less refined mesh. The run was then repeated with significantly more mesh elements placed near the tip. The total number of mesh elements for the initial and final states ranged approximately from 6000 to 200000 . For mesh B, the number of elements positioned adjacent to the interface was directly prescribed, as was the ratio of the edge length of the smallest element adjacent to the conic tip to that of the largest element adjacent to the outermost domain boundary. The mesh was then iteratively refined by splitting elements in the vicinity of the tip, as with mesh A. The number of elements for mesh B for the initial and final states ranged from approximately 30000 to 70000 .

Time integration was conducted using a variable first or second order backwards differentiation scheme with adaptive time stepping for a total of about 1000 time steps per simulation. Simulations were allowed to run just until interface acceleration near the tip led to irreparable mesh inversion. In all cases, the results reported in this work reflect simulations that were all terminated when the capillary pressure at the conic tip reached a given specified value. This was enforced by stopping runs when the dimensionless radius of curvature at the conic tip (scaled by $h_{o}$ ) attained the value $2.722 \times 10^{-4}$. In what follows, the final time at which this point was achieved is denoted by $T_{f}$. This cutoff length scale typically corresponded to a length ten times smaller than the minimum mesh edge length along the moving interface. In previous work modeling specific liquid metals, ${ }^{24,25}$ researchers have instead terminated simulations once the electric field strength at the conic apex was found to exceed the field emission value.

\section{RESULTS}

The following simulations represent results obtained for the Reynolds number ranging from $0.1 \leq \mathrm{Re} \leq 5 \times 10^{4}$ at fixed capillary number $\mathrm{Ca}=7.0834$. Details of the evolving interface shape and exponents characterizing the divergent behavior of governing forces at the conic tip are discussed next.

\section{A. Self-sharpening of conic cusp}

Shown in Figs. 3(a)-3(d) are far field views of the dimensionless interface shape $H(R, T)$ for values of Re spanning the viscous to inertial regime. For each value $\mathrm{Re}$ shown, the two curves depicted in red in (a)-(d) bracket the time interval observed to undergo selfsimilar growth, to be discussed in more detail below. The final curves shown in (a)-(h) correspond to the final simulation time $T_{f}$ when the dimensionless radius of curvature at the conic tip (scaled by $h_{0}$ ) attained the specified value $2.722 \times 10^{-4}$. Due to viscous retardation effects, it is evident that the time interval required to attain this small radius of curvature at $\mathrm{Re}=0.1$ is approximately two orders of magnitude longer than the time required at higher Reynolds numbers.
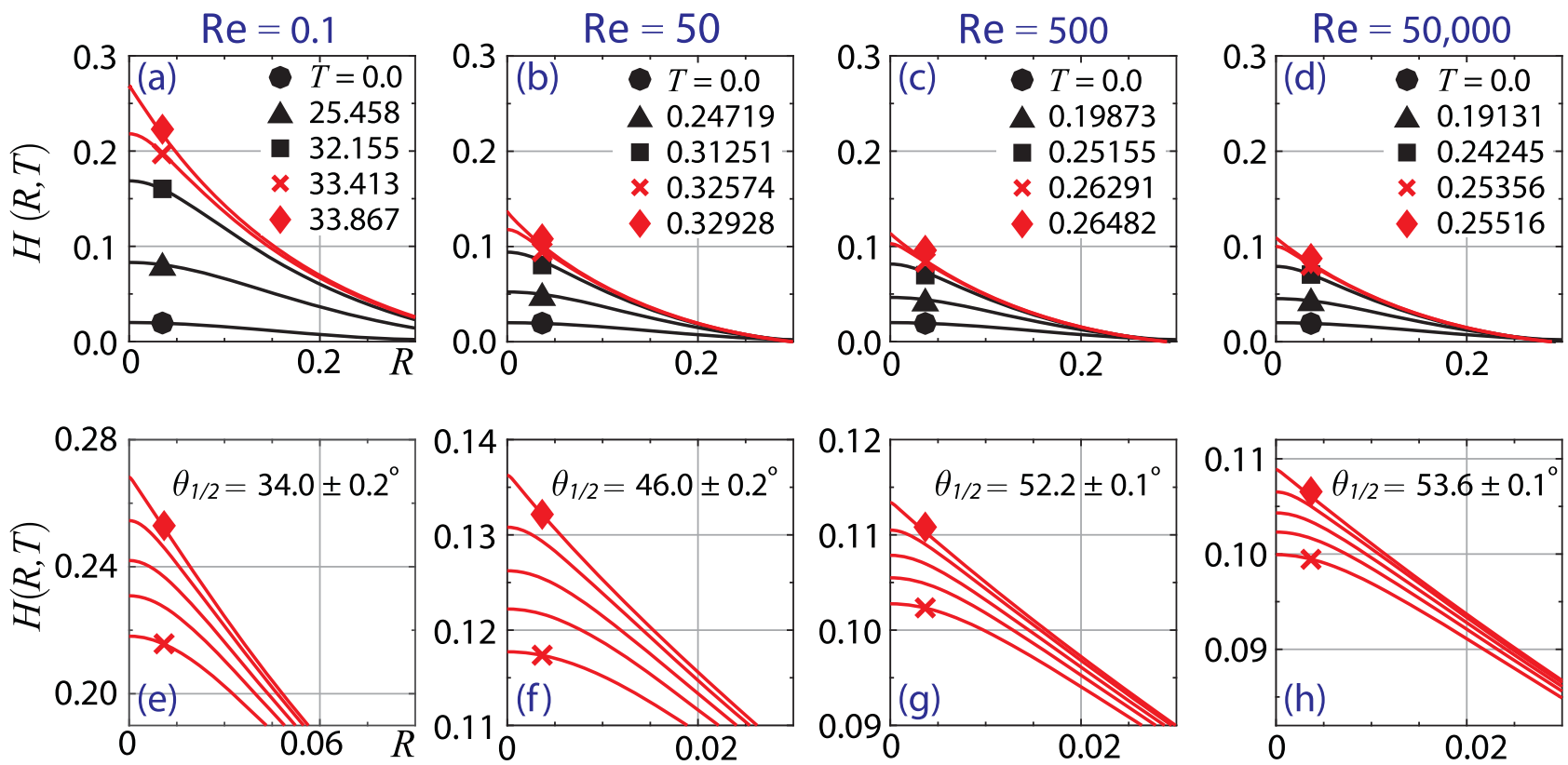

FIG. 3. Far field (upper panel) and magnified (lower panel) images (aspect ratio 1:1) of dimensionless interface shapes $H(R, T)$ for $R e=0.1,50,500,50000$ at $C a=7.0834$ (a)-(d) Snapshots of axisymmetric interface shapes for $0 \leq T \leq T_{f}$ where $T_{f}$ denotes the simulation final time when the dimensionless apical radius of curvature (scaled by $\left.h_{0}\right)=2.722 \times 10^{-4}$. The two uppermost curves (red) bracket the interval in time used to extract self-similar exponents. (e)-(h) Magnified views of the conic tip showing intermediate shapes for time interval indicated by the red curves in (a)-(d). Time stamps for intermediate curves are as follows: Re $=0.1, T=33.593,33.713,33.809$; $\operatorname{Re}=50, T=0.32710,0.32804,0.32882 ; \operatorname{Re}=500, T=0.26364,0.26415,0.26457 ; \operatorname{Re}=50000, T=0.25418,0.25460,0.25495$. Quantity $\theta_{1 / 2}$ represents the interior half-angle value at $T=T_{f}$ obtained from linear regression over the interval $0.005 \leq R \leq 0.030$. 
The dimensionless peak amplitudes in Fig. 3 also decrease in magnitude with increasing Re. This behavior can be understood from the discussion in Sec. III B 1 where it is shown that an increase in $\mathrm{Re}$ by a factor $\mathrm{f}$ at fixed $\mathrm{Ca}$ leads to an increase in the system size from $h_{o}$ to $f^{2} h_{o}$, modulo the constraint that the peak height was terminated once the apex radius of curvature achieved the value $2.722 \times 10^{-4} h_{o}$.

Shown in Figs. 3(e)-3(h) are magnified images of the evolving tip during the period of self-similar growth along with the values of the interior half-angle $\theta_{1 / 2}$ obtained from linear regression of $H\left(R, T_{f}\right)$ over the range $0.005 \leq R \leq 0.030$. (Since $H$ and $R$ are both scaled by $h_{o}$, this angular value is a constant whether viewed in dimensional or nondimensional coordinates.) These simulations confirm very rapid growth of conic tips with interior half-angle values above and below the static Taylor cone value of $49.3^{\circ}$. Larger values of $\mathrm{Re}$ are characterized by larger values of $\theta_{1 / 2}$. This can be understood from the fact that at a fixed capillary number, an increase in Reynolds number from $R e$ to fRe leads to a decrease in the initial electric field strength from $E_{c}$ to $E_{c} / f$. Weaker Maxwell forces lead to growth of wider cones, as expected.

\section{B. Velocity and strain rate behavior near the conic apex}

Shown in Fig. 4 are close-up images of the liquid tip at $T_{f}$ for $R e=0.1,50,500,50000$ at $\mathrm{Ca}=7.0834$. These images are color coded by the magnitude of the dimensionless velocity $|\boldsymbol{U}|$. Scale bars are marked as shown in the lower left corner of each
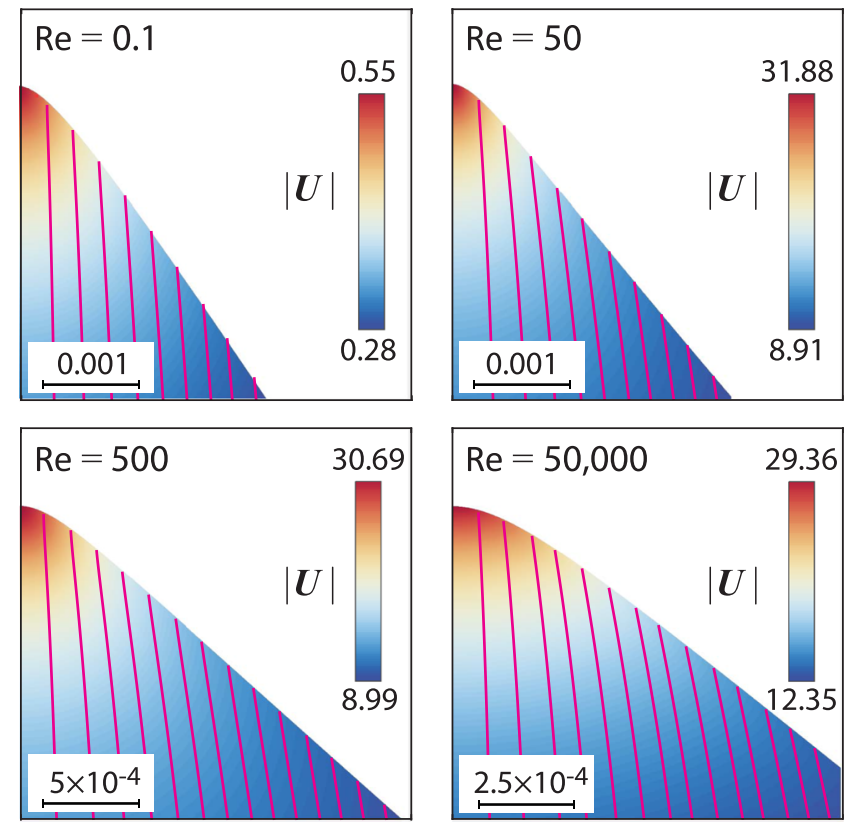

FIG. 4. Color map of the conic tip showing the magnitude of the (dimensionless) velocity field at $T=T_{f}$ for $\operatorname{Re}=0.1,50,500,50000$ at $\mathrm{Ca}=7.0834$. Scale bar on the lower left corner of each image indicates the dimensionless length (scaled by $h_{0}$ ). The vertical color stripe indicates the minimum and maximum flow speeds attained in the image shown. Velocity streamlines (magenta) are superimposed on the liquid domain.
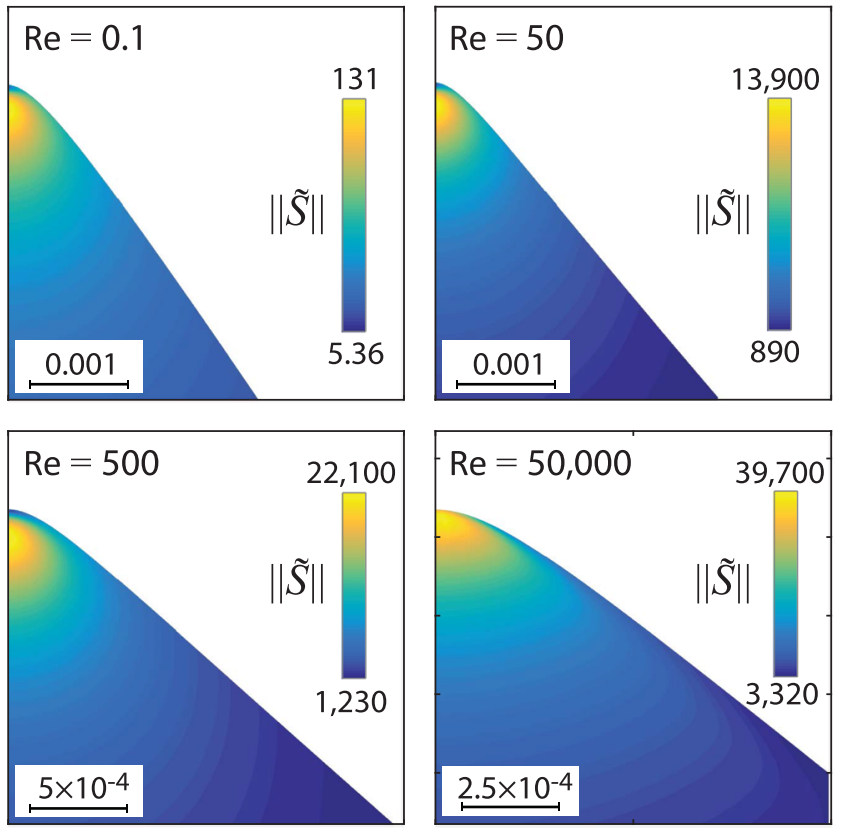

FIG. 5. Color map of the conic tip showing the magnitude of the (dimensionless) rate of strain field $\|\widetilde{\boldsymbol{S}}\|$ at $T=T_{f}$ for $\operatorname{Re}=0.1,50,500,50000$ at $\mathrm{Ca}=7.0834$. Scale bar on the lower left corner of each image indicates the dimensionless length (scaled by $h_{0}$ ). The vertical color stripe indicates the minimum and maximum rates of strain attained in the image shown.

image. The curves superimposed on the liquid domain represent the velocity streamlines, which indicate that the net flow to the liquid tip for all $\mathrm{Re}$ is mostly oriented in the vertical direction (in contrast to Zubarev's inviscid analysis, ${ }^{20}$ which predicts a radial sink flow toward the apex point). The color maps also indicate that the highest flow speeds are concentrated near the accelerating tip, as expected. However, while the maximum velocity undergoes a significant increase from $R e=0.1$ to $R e=50$, there is relatively little change in the maximum value beyond that. This is due to the fact that the simulations were all terminated once the apex radius of curvature attained the dimensionless value $2.722 \times 10^{-4}$, which essentially established the same maximum attainable (dimensionless) values for the local capillary pressure and corresponding fluid velocity.

Shown in Fig. 5 are the same set of close-up images as Fig. 4, now colored by the magnitude of the dimensionless rate of strain tensor $\|\widetilde{\boldsymbol{S}}\|=\left(\widetilde{\boldsymbol{S}}: \widetilde{\boldsymbol{S}}^{T}\right)^{1 / 2} / \sqrt{2}$, where $\widetilde{\boldsymbol{S}}=\left[\widetilde{\nabla} \boldsymbol{U}+(\widetilde{\nabla} \boldsymbol{U})^{T}\right] / 2$. The region of the largest strain rate was observed to occur just below the surface of the conic tip. This region, which sustains the largest rate of deformation, undergoes the highest acceleration. The extent of this region of the highest strain rate was observed to diminish in width for increasing Reynolds number.

The very large strain rates observed in the vicinity of the accelerating conic tip give rise to an interfacial boundary layer. Examination of the vertical velocity component along the axis of symmetry $R=0$ reveals its behavior with increasing $\mathrm{Re}$. Shown in Fig. 6 are curves of the normalized velocity $V\left(R=0, Z, T_{f}\right) / V_{f}^{\text {tip }}$ vs 


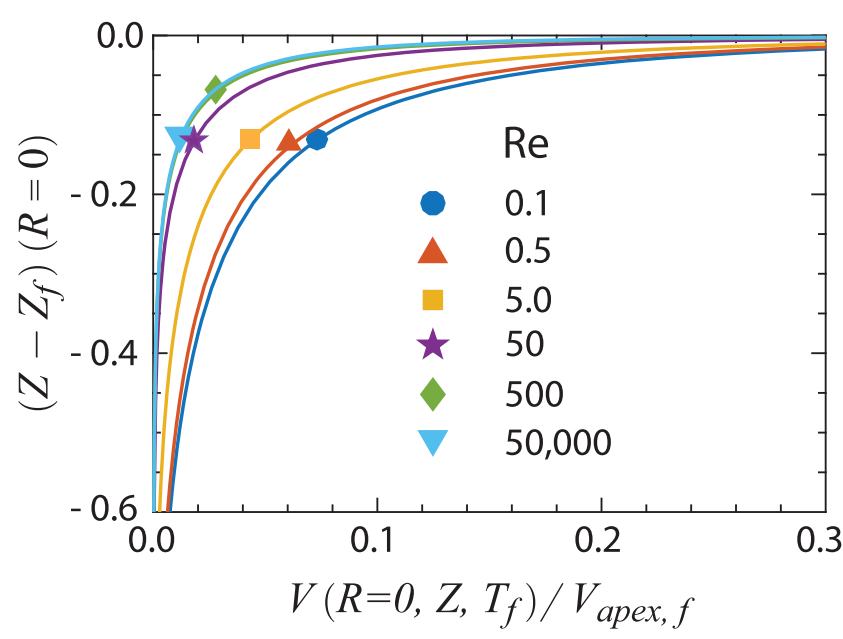

FIG. 6. Dimensionless velocity ratio $V\left(R=0, Z, T_{f}\right) / V_{\text {apex } f}$ vs dimensionless distance $Z-Z_{f}(R=0)$ for increasing $\mathrm{Re}$ at $\mathrm{Ca}=7.0834$, where $Z_{f}$ denotes the apex vertical coordinate at $T_{f}$ and $V_{\text {apex }, f}=V\left(R=0, Z_{f}, T_{f}\right)$ denotes the corresponding vertical flow speed.

distance along the central axis $Z-Z_{F}(R=0)$ for increasing Re where $V_{F}^{\text {tip }}=V\left(R=0, Z_{f}, T_{f}\right), T_{f}$ denotes the final simulation time, and $Z_{f}(R=0)$ is the vertical coordinate of the conic apex at time $T_{f}$. As evident, the boundary layer thickness $\delta_{a v g}$ decreases with increasing $\operatorname{Re}$ however not according to the relation $\delta_{\text {avg }} \sim \operatorname{Re}^{-1 / 2}$, as in the simple Blasius problem ${ }^{37}$ where a solid substrate moving at constant speed causes motion in the adjacent liquid. In the current problem, the vacuum/liquid interface is a shear free surface that undergoes nonuniform acceleration due to the spatially dependent Maxwell stresses acting along the surface. Further study of this boundary layer is ongoing.

Due to axisymmetry, the quantity $\|\widetilde{S}\|(R=0, Z)$ reduces simply to the value $\sqrt{2}|\partial V / \partial Z|(R=0, Z)$. Therefore, the regions of the highest strain rate in Fig. 5 correspond to the uppermost portions of the boundary layer where $\partial V / \partial Z$ is largest. Inspection of Fig. 6 further reveals that the thickness of the boundary layers is much larger than the vertical displacement in the corresponding interface shapes in Fig. 5. The regions of the highest strain rate are therefore all nestled deep within the boundary layer, which extends much further into the bulk liquid.

\section{Self-similar evolution of conic tip}

We now turn to the evidence of self-similar growth for all Reynolds numbers spanning the viscous to inviscid regime. The magnitudes of the various forces per unit area and forces per unit volume acting at the conic apex, evaluated from the normal stress boundary condition in Eq. (52) and the Navier-Stokes equation in Eq. (51), are shown to sustain extended self-similar growth upon approach to the blowup time. Self-similar variable transformations obtained from exponents extracted from the numerical simulations conducted at various $\mathrm{Re}$ at fixed $\mathrm{Ca}$ confirm that the accelerating liquid tip undergoes collapse toward a conic shape whose interior angle increases with increasing $\mathrm{Re}$.

\section{Determination of blowup time $T_{B}$ and blowup apex coordinate $Z_{B}$}

In order to extract accurate power law exponents characterizing blowup behavior in the forces acting at the conic apex, it was first necessary to estimate from the simulations the (virtual) asymptotic blowup time $T_{\mathrm{B}}$. For each run at fixed Re, this time was estimated by tracking the growth in the Maxwell pressure at the conic apex as $\tau=T_{\mathrm{B}}-T \rightarrow 0$ (plotted on double logarithmic axes) and then choosing the value $T_{\mathrm{B}}$ that produced the best linear fit over the final two and a half decades in $\tau$ as $\tau \rightarrow 0$ [indicated by the vertical dashed lines in Figs. 7(a)-7(h)]. Similarly, the (virtual) asymptotic vertical coordinate for the apex height $Z_{\mathrm{B}}$ was estimated by tracking the advance of the vertical coordinate of the conic apex as $\tau \rightarrow 0$ (plotted on double logarithmic axes) and then choosing the value $Z_{\mathrm{B}}$ that produced the best linear fit over the final two and a half decades in $\tau$ as $\tau \rightarrow 0$ for the range $0.005 \leq R \leq 0.030$. All estimated values $T_{\mathrm{B}}$ and $Z_{\mathrm{B}}$ are listed in Table II.

\section{Exponents describing self-similar growth of normal stresses at conic apex}

Shown in Figs. $7(\mathrm{a})-7(\mathrm{~d})$ are representative plots showing the magnitude of the Maxwell $(M)$, capillary $(C)$, and viscous $(V)$ normal stresses (i.e., normal forces per unit area) acting at the conic apex for increasing $\mathrm{Re}$ at $\mathrm{Ca}=7.0834$, as computed from the terms in Eq. (52). The power law exponents $\beta_{M}, \beta_{C}$, and $\beta_{V}$ were extracted from the growth behavior during the final two and a half decades in time as $\tau \rightarrow 0$. The results indicate that all three normal stresses diverge as $\tau^{-\beta_{j}}$, where $j=M, C$, and $V$. Listed in Table II are the complete set of exponents and fitting parameters for all simulations in this study. In general, $\beta_{M}$ and $\beta_{C}$ tend to decrease with increasing $\mathrm{Re}$, suggestive of stronger divergent behavior at a higher Reynolds number.

As evident in Figs. 7(a)-7(d), the Maxwell stress magnitude at the conic apex always exceeds that of the capillary and viscous normal stress irrespective of the Reynolds number. At small values of $\mathrm{Re}$, the Maxwell pressure exceeds the capillary and viscous normal stress by at least an order of magnitude. As Re increases, the Maxwell and capillary pressures approach each other in magnitude, while the viscous normal stress decreases by several orders of magnitude. This trend is understandable since viscous effects proportionally diminish in magnitude as Re increases. Furthermore, while at small Re the difference in pressure between the Maxwell and the capillary stress increases as $\tau \rightarrow 0$, this difference becomes smaller with increasing $\mathrm{Re}$ as the blowup time is approached.

In evaluating the actual sign of these normal stresses, it is clear from the magnified images in Figs. 4 and 5 that the liquid shape near the apex is given by a convex curve. The capillary stress in Figs. 7(a)-7(d) is therefore always a positive quantity for all Reynolds numbers. In our study, the capillary pressure always represses tip sharpening, but it is always overcome by the Maxwell pressure, which is a negative quantity. Both pressures increase in magnitude as the apex radius of curvature decreases in time. This apical selfsharpening goes hand in hand with the local self-enhancement of the electric field in that region. Were it the case that the electric field at the tip was not governed by its local value but instead by the global background value set by the external field, then self-similar growth would not be possible since the relative length and time scales could 
$\operatorname{Re}=0.1$
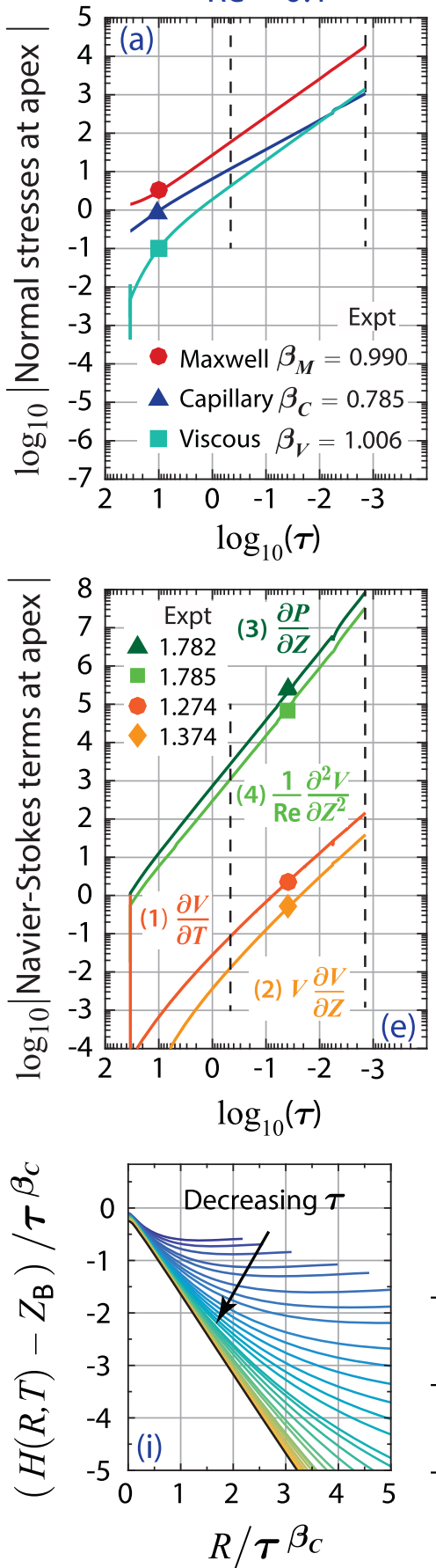

$\operatorname{Re}=50$
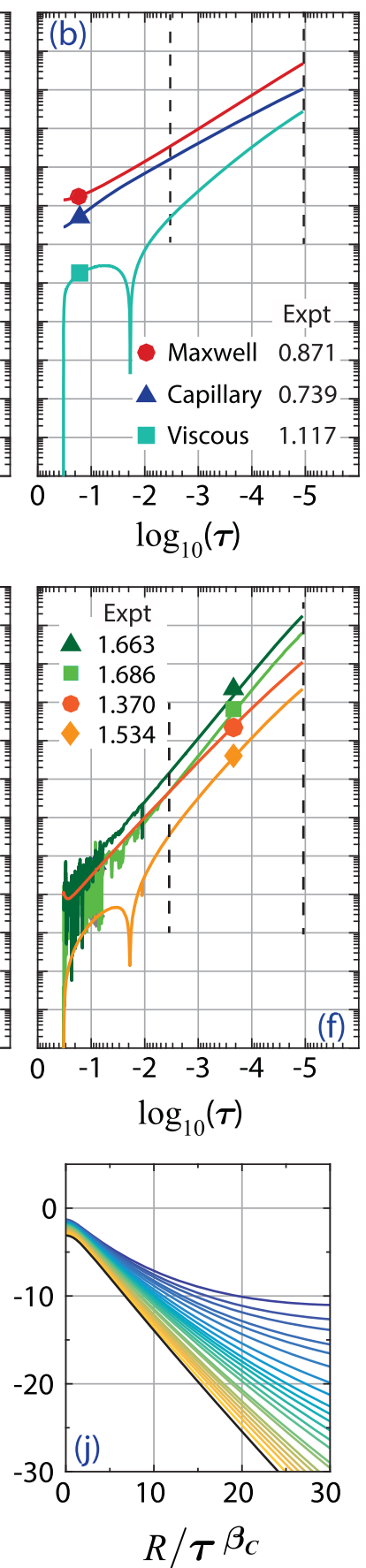

$\operatorname{Re}=500$
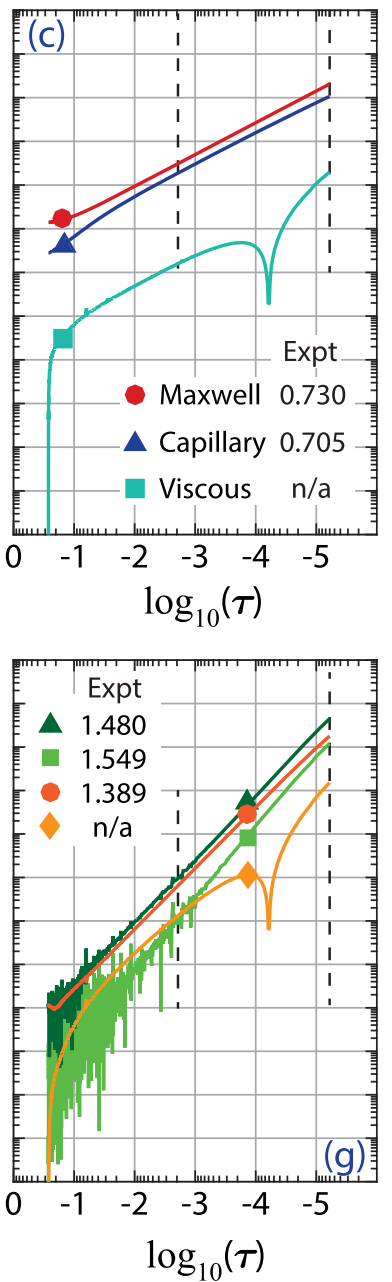

(g)

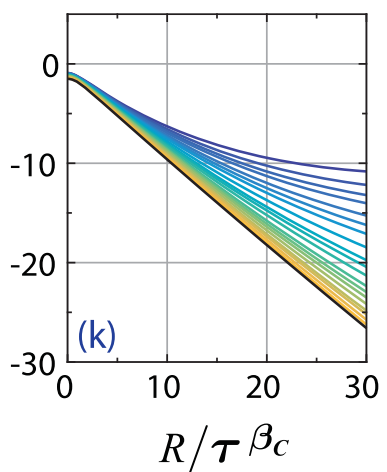

$\operatorname{Re}=50,000$

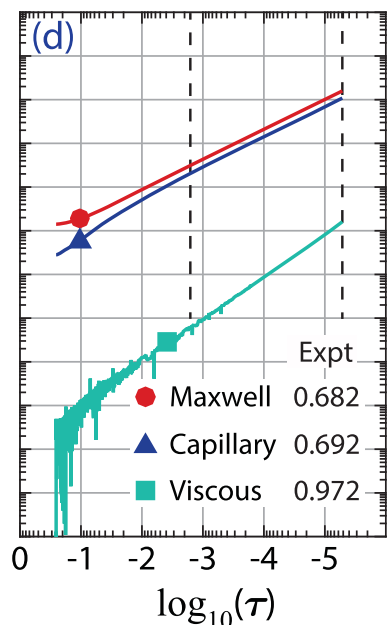

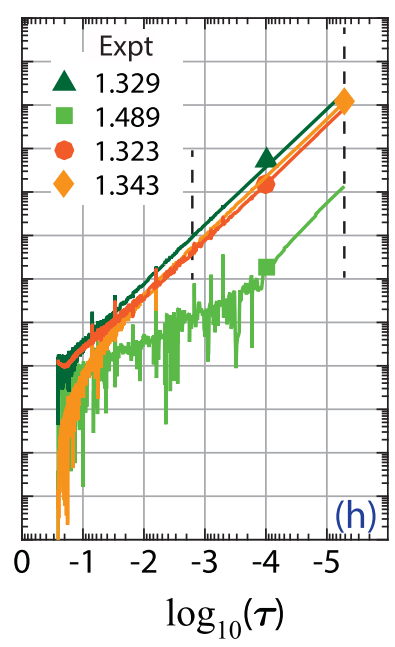

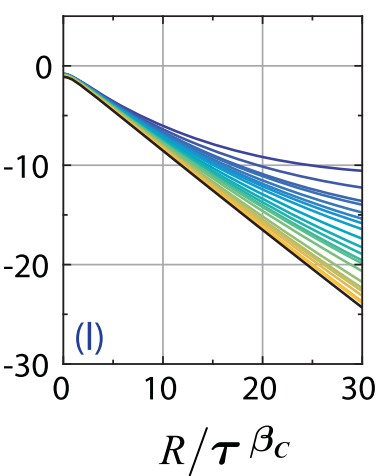

FIG. 7. [(a)-(h)] Self-similar growth of forces acting at the conic apex with corresponding power law exponents. Table II contains a complete listing of all fitted exponents (to higher precision than listed in the images). [(a)-(d)] Double logarithmic plots showing the absolute value of the Maxwell, capillary, and viscous normal stresses (forces/unit area) acting at the conic apex $\left(R=0, Z=H_{\text {apex }}\right)$ vs $\tau=T_{B}-T$, as computed from Eq. (52). Exponent values for $\beta_{M}, \beta_{C}$, and $\beta_{V}$ were obtained by linear regression over the interval indicated by the vertical dashed lines. [(e)-(h)] Double logarithmic plots showing the absolute value of the forces per unit volume acting at the conic apex vs $\tau$ $=T_{\mathrm{B}}-T$, as computed from Eq. (54). Exponent values for terms (1)-(3) (i.e., $\beta_{1}$ through $\beta_{3}$ ) were obtained by linear regression over the interval marked by the vertical dashed lines except for term 4 for $\operatorname{Re}>200$ for which the fits were only carried out over the final decade in $\tau$. [(i)-(I)] Self-similar shape of conic cusp upon uniform rescaling of the horizontal and vertical coordinates by the factor $\tau^{-\beta_{C}}$ showing every fifth time step in $\tau$ for the period of self-similar growth indicated by the dashed vertical lines in (a)-(h). 


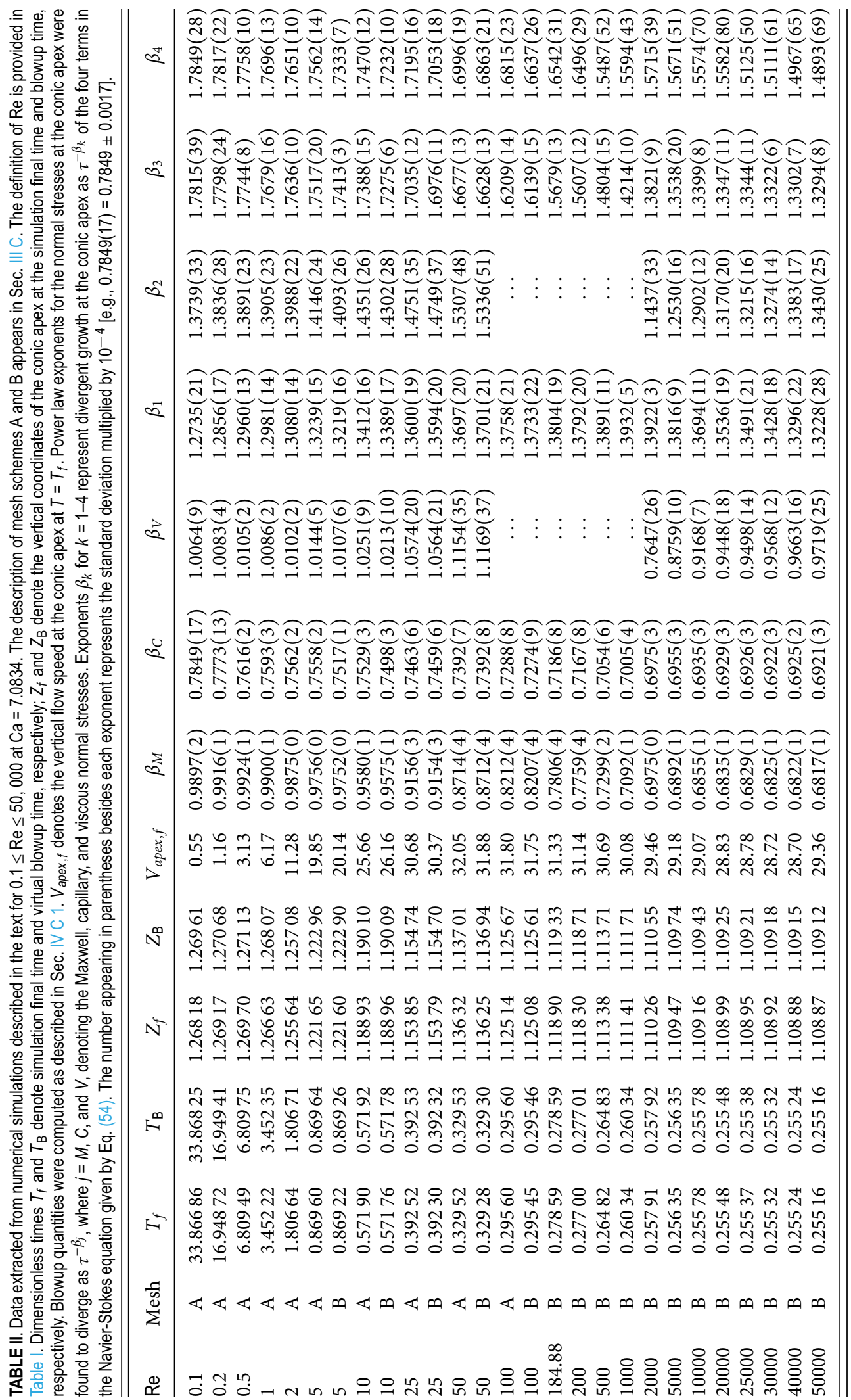


not conform to yield self-similar scalings. This insight that the apical field strength rapidly exceeds the background value is what motivated $\mathrm{Zubarev}^{20}$ to seek self-similar behavior in the apex region of the flow.

The viscous normal stress can assume both positive and negative values depending on $\operatorname{Re}$ and $\tau$. For example, the dip in the viscous normal stress in Fig. 7 (a) at early time near $\log _{10} \tau=-1.6$ is caused by a transition from positive to negative values. As $\tau \rightarrow$ 0 , the viscous normal stress becomes increasingly more negative. Inspection of the velocity gradient $\partial V / \partial Z$ near the tip along the axis of symmetry, indicated in Fig. 5, shows that the maximum in the vertical velocity component does not occur at the conic apex but instead at a short distance below-behavior that therefore signals that $\tau_{Z Z} \propto \partial V / \partial Z<0$ at the conic apex. We are currently exploring whether vorticity effects in the conic region are responsible for this small vertical displacement in the velocity maximum. Likewise in Fig. 7(b), the dip in viscous normal stress near $\log _{10} \tau$ $=-1.8$ signals a transition from positive to negative values. In Figs. 7 (a) and 7 (b), this transition occurs prior to the onset of the self-similar regime. In Fig. 7(c), however, this transition occurs within the self-similar period of growth, which therefore precludes extraction of a meaningful exponent-hence the designation "n/a." In such cases, we do not report in Table II exponent values $\beta_{V}$ for $100 \leq \operatorname{Re} \leq 1000$. For $\operatorname{Re}>1000$, the viscous normal stress is positive at all times, allowing extraction of $\beta_{V}$ from the indicated curve in Fig. 7(d) for $\operatorname{Re}=50000$.

\section{E. Exponents describing self-similar growth of Navier-Stokes terms at conic apex}

We next evaluate the temporal behavior of each term in the Navier-Stokes equation at $(R=0, Z=H)$, which for axisymmetric geometry, simplifies to the form

$$
\underbrace{\frac{\partial V}{\partial T}}_{\text {Term } 1}+\underbrace{V \frac{\partial V}{\partial Z}}_{2}=-\underbrace{\frac{\partial P}{\partial Z}}_{3}+\underbrace{\frac{1}{\operatorname{Re} \frac{\partial^{2} V}{\partial Z^{2}}}}_{4} .
$$

These four terms, which represent the forces per unit volume acting at the conic apex, describe fluid (1) acceleration, (2) advection, (3) pressure gradient, and (4) viscous forces. In what follows, the exponents associated with these terms are labeled $\beta_{1}$ to $\beta_{4}$. The plots shown in Figs. $7(\mathrm{e})-7(\mathrm{~h})$ confirm self-similar growth of the conic tip as $\tau \rightarrow 0$. As before, we do not report in Table II any exponent values $\beta_{4}$ for the dynamic range $100 \leq \mathrm{Re} \leq 1000$ due to the presence of the sharp dip in the viscous stress within the self-similar regime caused by a change in sign [e.g., Fig. $7(\mathrm{~g})$ ].

The results in Fig. $7(\mathrm{e})$ confirm that at a low Reynolds number such as $\operatorname{Re}=0.1$, the dominant competition is given by the pressure gradient and the viscous term, both roughly five orders of magnitude larger than the inertial terms given by terms 1 and 2 . This difference in magnitude between terms $3-4$ and $1-2$ is observed to increase as $\tau \rightarrow 0$. As Re increases, however, the inertial terms 1 and 2 become more prominent, eventually approaching similar magnitudes as the pressure gradient. By contrast, the viscous term decreases in magnitude, becoming rather negligible by about $R e=20000-30000$ (not shown). For $R e=50000$, it is evident from Fig. 7(h) that the viscous normal stress at the conic tip is at least two orders of magnitude smaller than the other three terms in the Navier-Stokes equation. In all the simulations conducted, the magnitude of the pressure gradient given by term 3 exceeded that of the other terms throughout the period governed by self-similar growth. This likely reflects the fact that the rapidly increasing Maxwell pressure at the conic apex remains the dominant factor in the pressure gradient irrespective of the Reynolds number.

For completeness, we note here the changes in sign that occur in time for terms 1-4 in Eq. (54) and shown in Figs. 7(e)-7(h). Term 1 , which tracks the acceleration of the conic apex, is always positive irrespective of the value of $\mathrm{Re}$. This must be the case since the dominant driving force at the tip is the Maxwell pressure that increases rapidly in time due to self-enhancement effects. Term 2 tracks the inertial advective term whose sign depends on $\operatorname{Re}$ and $\tau$. For $\operatorname{Re}=0.01$, the actual sign of term 2 is negative, which implies that $\partial V / \partial Z<0$ at the apex. For $\operatorname{Re}=50$ and 500 , there occurs a sharp dip in term 2, which signals a transition from positive to negative values as $\tau$ decreases. For $\operatorname{Re}=50000$, there initially occurs significant scatter in term 2 which eventually settles down to reveal steady power law growth. During this latter period, term 2 is always positive in value.

Except for some scatter at early times, term 3 given by the pressure gradient $\partial P / \partial Z$ is always negative irrespective of the Reynolds number, which indicates that the driving pressure gradient $\partial P / \partial Z>0$. This behavior is expected since the dominant driving force due to the Maxwell pressure at the conic apex always pulls fluid toward the counter electrode. The viscous force per unit volume given by term 4 is well behaved at a small Reynolds number and always negative, counterbalancing the pressure gradient term. The data for term 4 become significantly noisier with increasing Re, while its magnitude decreases significantly, especially within the period of self-similar growth. This behavior can be traced to the rapid change in the vertical velocity component along the central axis within a distance of a few mesh elements of the accelerating interface. For $\operatorname{Re}=50$ and 500, this noisy regime occurs prior to the time interval used to extract the corresponding exponent. For $\mathrm{Re}=50000$, this noisy behavior persists well into the self-similar regime. For this reason, the exponents reported for term 4 for $\operatorname{Re}>200$ were extracted using only data generated during the final decade in time where there occurs smooth power law growth.

\section{F. Universal self-similar shape of conic tip}

The time sequence images in Figs. 7(i)-7(1) confirm self-similar collapse of interface shapes obtained by rescaling the horizontal and vertical coordinates by the factor $\tau^{-\beta_{C}}$, where $\beta_{C}$ is the blowup exponent for the capillary pressure plotted in Figs. 7(a)-7(d). This dynamic length was chosen for the rescaling of the interface since it is a direct measure of the diminishing radius of curvature at the conic apex as time proceeds. The curves in Figs. 7(i)-7(1) depict the evolving interface shapes during the period of self-similar growth identified in (a)-(h). The nonuniform spacing between curves reflects the adaptive time stepping used in the simulations, as discussed in Sec. III C.

\section{G. Variation of normal stress exponents with increasing $\mathrm{Re}$}

The simulations conducted in this study, whose range in Reynolds number spans the viscous to the inertial regime, 
confirm self-similar growth of the conic tip irrespective of the Reynolds number. As clear from Figs. 7(a)-7(h), the pairs of dominant competitive forces that are responsible for self-similar blowup change as $\mathrm{Re}$ increases. Figure 8 illustrates the variation in the magnitude of the blowup exponents $\beta_{M}, \beta_{C}$, and $\beta_{V}$ with increasing $\mathrm{Re}$ at $\mathrm{Ca}=7.0834$. The dashed horizontal lines represent the exponent values first predicted by Zubarev ${ }^{20}$ for conic cusp growth in the inviscid limit and by Fontelos et $a l^{34}$ in the Stokes limit. Error bars, which were always smaller than $10^{-3}$, are not shown since they are smaller in size than the icons designating the mean values. Included in Fig. 8 are the results for $\operatorname{Re}=5,10,25,50$, and 100 obtained from the two meshing schemes (A and B) discussed in Sec. III C. These data are nearly indistinguishable, confirming that the two meshing schemes produced virtually identical results.

Figure 8 illustrates which normal stresses at the moving interface maintain similar exponents. At the smallest values of $\mathrm{Re}$, the Maxwell and viscous normal stresses (i.e., forces per unit area) assume a value close to one, although as indicated in Fig. 7(a), their actual magnitudes differ by more than an order of magnitude. As is perhaps more evident from Fig. 7(e), which shows the resulting forces per unit volume acting at the tip, the Maxwell and viscous forces provide the main competitive balance throughout the viscous dominated regime, while the capillary force per unit volume acts more as a smaller corrective term. By contrast, at much higher values of the Reynolds number, the dominant force balance transitions to that between the Maxwell and the capillary stress, while the viscous normal stress appears as a smaller correction. These results confirm the dominant force balance reported by Fontelos et al. in the Stokes limit ${ }^{34}$ and $\mathrm{Zubarev}^{20}$ in the inviscid limit.

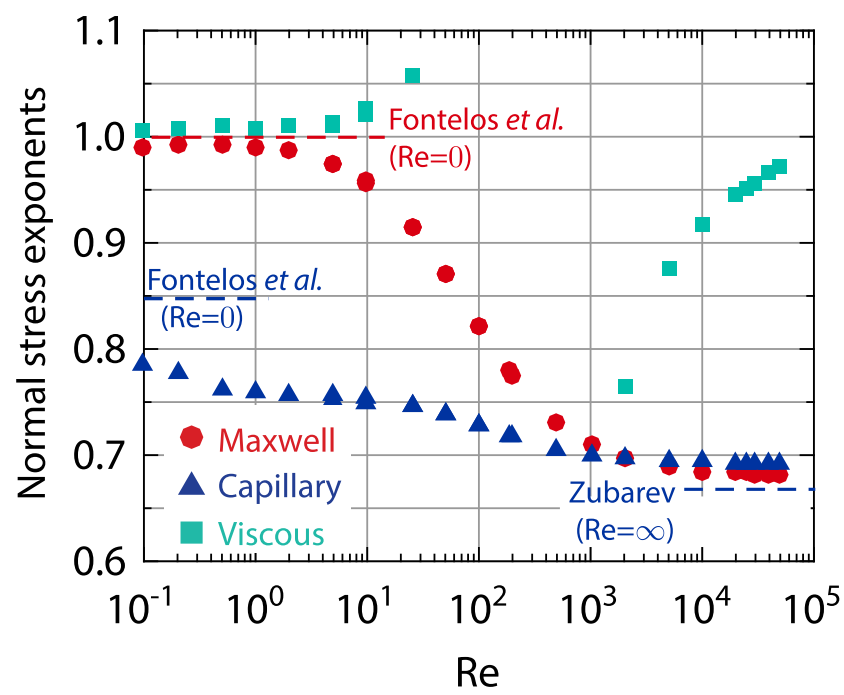

FIG. 8. Semilogarithmic plot of the magnitude of the exponents $\beta_{M}, \beta_{C}$, and $\beta_{V}$ vs $\operatorname{Re}$ computed from the terms in the normal stress boundary condition given by Eq. (52). Superimposed dashed lines indicate the asymptotic values predicted by Fontelos et al..$^{34}$ and by Zubarev, ${ }^{20}$ as discussed in Sec. IV H. The complete set of exponents are listed in Table II.

\section{H. Comparison to asymptotic exponents predicted by Zubarev and Fontelos et al.}

The results in Fig. 8 for the exponents $\beta_{M}$ and $\beta_{C}$ indicate that as the Reynolds number increases toward the inviscid limit, both exponents asymptote toward the value $2 / 3$, which Zubarev ${ }^{20}$ surmised from the scaling invariances discussed in Sec. II B 2. Zubarev's scalings in Eqs. (13)-(16) can also be used to estimate the corresponding asymptotic exponent for the viscous normal stress. Noting that the viscous normal stress in Eq. (52) at the apex is given by $\partial V / \partial Z$, it must therefore scale as $\tau^{-\beta_{V}}=\tau^{-1}$. Our simulations confirm that at $\mathrm{Re}=50000$, the magnitude of this exponent has already reached a value $0.9719 \pm 0.0025$, close to the asymptotic limit of one. The trend evident from Fig. 8 is that the magnitude of $\beta_{V}$ will further smoothly increase toward unity as $\operatorname{Re} \rightarrow \infty$.

Zubarev's self-similar analysis requires that spatial dimensions scale as $\tau^{2 / 3}$, flow velocities scale as $\tau^{-1 / 3}$, and the capillary and Maxwell pressures scale as $\tau^{-2 / 3}$. In concluding the analysis in his original work, he applied these scalings from his inviscid model to the Navier-Stokes equation in Eq. (54) and found that

$$
\begin{gathered}
\underbrace{\frac{\partial V}{\partial T} \sim \underbrace{V \frac{\partial V}{\partial Z}}_{2} \sim \underbrace{\frac{\partial P}{\partial Z}}_{3} \sim \tau^{-4 / 3},}_{1} \\
\underbrace{\frac{1}{\operatorname{Re} \frac{\partial^{2} V}{\partial Z^{2}}}}_{4} \sim \tau^{-5 / 3} .
\end{gathered}
$$

Inspection of the exponent values in Table II for $\mathrm{Re}=50000$ from our simulations, namely, $\beta_{1}=1.3228 \pm 0.0028, \beta_{2}=1.3430 \pm 0.0025$, and $\beta_{3}=1.3294 \pm 0.0008$, confirms close agreement with the inviscid prediction of $4 / 3$. Our value $\beta_{4}=1.4893 \pm 0.0069$ for the viscous stress is somewhat smaller than his prediction of $5 / 3$.

It is worth noting here that although Zubarev's findings stem from a self-similar analysis of Bernoulli's equation under the assumption of inviscid and irrotational flow, the same exponents can also be obtained from the Euler equation (i.e., the inviscid limit of the Navier-Stokes equation) and the corresponding inviscid limit of the normal stress boundary condition. A back of the envelope calculation leads to the prediction

$$
\beta_{M}=\beta_{C}=2 / 3 \text { and } \beta_{V}=1 .
$$

Apparently then, the irrotational constraint inherent in the Bernoulli analysis appears not to be a necessary requirement for the values obtained for these exponents. Indeed, in all our studies, we have observed the presence of interface generated vorticity concentrated just below the accelerating interface, which we are investigating in a separate work.

The results of our simulations also show good agreement with the predictions of Fontelos et $a l^{34}$ for $\operatorname{Re}=0$. As discussed in Sec. II D, these researchers demonstrated that at $\operatorname{Re}=0$, the electric field strength at the apex of an electrified liquid drop must diverge as $\tau^{-1 / 2}$ or, equivalently, that the Maxwell pressure must scale as $\tau^{-1}$, which leads to a value $\beta_{M}=1$. Our simulations at $\operatorname{Re}=0.1$ yield exponent values $\beta_{M}=0.9897 \pm 0.0002$, in excellent agreement with their asymptotic prediction. Their analysis also predicts that the exponent associated with the capillary pressure must vary with the cone interior half-angle $\theta_{1 / 2}$ such that $\beta_{C}=\alpha\left(\theta_{1 / 2}\right)$. In Ref. 34, 
the authors provide a numerical plot detailing the variation in exponent values corresponding to the flow velocity (and electric potential) as a function of increasing cone interior half-angle. From that plot and the value of the interior half-angle $\theta_{1 / 2}=34^{\circ}$ extracted from our Fig. 3(e) for $\operatorname{Re}=0.1$, we determined that $\alpha=0.8465$. Our estimated value of $\beta_{C}=0.7849 \pm 0.0017$ for $\operatorname{Re}=0.1$, which according to the trend shown in Fig. 8 will increase as the Reynolds number is lowered still, is in good agreement with their prediction $\beta_{C}=0.8465$.

Noting once again that the viscous normal stress in Eq. (52) at the apex is given by $\partial V / \partial Z$, it must then be the case from the scalings for $V$ and $Z$ that the viscous normal stress scales as $\tau^{-1}$, the same scaling obtained in the limit of high Re. Our simulations at $\operatorname{Re}=0.1$ yield a value $\beta_{V}=1.0064 \pm 0.0009$, in excellent agreement with this prediction. In fact, the dimensional analysis requires that $\partial V / \partial Z \sim \tau^{-1}$ regardless of the Reynolds number. Inspection of the values extracted for $\beta_{V}$ given in Table II indeed confirms that all the reported values are close to one. The largest deviations from unity occur for those intermediate values of Reynolds numbers, which as we noted earlier, incur a change in sign within the fitting interval and a corresponding sharp dip, as shown in Figs. 7(f)-7(h). The occurrence of these dips tends to skew the extracted values of $\beta_{V}$ slightly away from one.

The analysis by Fontelos et al. ${ }^{34}$ also predicts that $Z$ scales in time as $\tau^{\alpha}$ where $\alpha>0, V$ must scale as $\tau^{\alpha-1}$, and the voltage potential must scale as $\tau^{\alpha-1 / 2}$. The latter relation therefore requires that the electric field strength diverge as $\tau^{-1 / 2}$ and therefore yields that the Maxwell pressure must diverge as $\tau^{-1}$. Substituting these scalings into Eq. (54) for the value $\alpha=0.8465$ determined above for runs conducted at $\operatorname{Re}=0.1$ yields the following exponent values:

$$
\begin{gathered}
\frac{\partial V}{\partial T} \sim \tau^{\alpha-2} \approx \tau^{-1.1535}, \\
V \frac{\partial V}{\partial Z} \sim \tau^{\alpha-2} \approx \tau^{-1.1535}, \\
\frac{\partial P}{\partial Z} \sim \tau^{-1-\alpha} \approx \tau^{-1.8465}, \\
\frac{1}{\operatorname{Re}} \frac{\partial^{2} V}{\partial Z^{2}} \sim \tau^{-1-\alpha} \approx \tau^{-1.8465} .
\end{gathered}
$$

Listed in Table II are the values of the corresponding exponents extracted from the simulations run at $\operatorname{Re}=0.1$, namely, $\beta_{1}=1.2735$ $\pm 0.0021, \beta_{2}=1.3739 \pm 0.0033, \beta_{3}=1.7815 \pm 0.0039$, and $\beta_{4}=1.7849$ \pm 0.0028 , which are all consistent with those of Fontelos et al. in the limit $\operatorname{Re} \rightarrow 0$.

\section{DISCUSSION}

\section{A. Confirmation of laminar flow at conic tip}

The parameter range investigated in our study for which $0.1 \leq \mathrm{Re} \leq 50000$ at $\mathrm{Ca}=7.0834$ was confirmed to maintain laminar flow conditions throughout. While the Reynolds number defined in Table I is useful in cataloging the general flow behavior discussed, that value is based on the initial electric field strength $\phi_{o} / h_{o}$ and therefore represents a minimal value. In order to assess flow conditions in the region closest to the rapidly accelerating tip, it proves useful as well to define a local Reynolds number defined by

$$
\operatorname{Re}_{\text {apex }}=\frac{\rho v_{\text {apex }} r_{\text {apex }}}{\mu},
$$

where $v_{\text {apex }}$ denotes the dimensional vertical component of the fluid velocity at the conic apex and $r_{\text {apex }}$ denotes the apex radius of curvature. Accordingly, the local Reynolds number at the apex can be re-expressed as

$$
\operatorname{Re}_{\text {apex }}=V_{\text {apex }} R_{\text {apex }} \times \mathrm{Re} .
$$

Our simulations have shown that at a finite Reynolds number $\mathrm{Re}$, the capillary pressure at the apex scales as $\tau^{-\beta_{C}}$. Since this pressure is inversely proportional to the (dimensionless) apex radius of curvature $R_{\text {apex }}$, this implies that $R_{\text {apex }} \sim \tau^{\beta_{C}}$. Furthermore, since the viscous normal stress $\partial V / \partial Z$ at the apex scales as $\tau^{\beta_{V}}$, then $V \sim \tau^{\beta_{C}-\beta_{V}}$. These scalings therefore suggest that

$$
\operatorname{Re}_{\text {apex }} \sim \tau^{2 \beta_{C}-\beta_{V}} .
$$

For the exponent values $\beta_{C}$ and $\beta_{V}$ listed in Table II, it is clear that $2 \beta_{C}-\beta_{V}>0$ and therefore $\operatorname{Re}_{\text {apex }} \sim \tau^{2 \beta_{C}-\beta_{V}}$ decreases as $\tau=T_{\mathrm{B}}-T$ decreases, as confirmed by the curve shown in Figs. 9(a) and 9(b). In both plots, $R_{\text {apex }}$ decreases monotonically as the blowup time is approached due to the sharpening of the conic tip (i.e., diminishing values of $R_{\text {apex }}$ ), while $V_{\text {apex }}$ increases monotonically toward the singular point. A comparison of the magnitudes in Figs. 9(a) and 9(b) also reveals an increase in $\mathrm{Re}_{\text {apex }}$ by roughly two orders of magnitude as $R e$ is increased by the same factor. This correlation seems evident from Fig. 9(c) where the product $V_{\text {apex }}^{\max } R_{\text {apex }}^{\max }$ remains fairly constant as $R e$ increases except at the smallest values of $R e$ for the viscous dominated regime.

We note in Fig. 9(a) that the maximum value $R e_{\text {apex }}^{\max }=53.68$, which is achieved at $\log _{10} \tau=-1.1441$ (i.e., $T=0.19307$ ), indicative of laminar flow. In Fig. 9(b), the maximum value $\operatorname{Re}_{\text {apex }}^{\max }$ $=5462.4$ achieved at $\log _{10} \tau=-1.1535$ (i.e., $T=0.18511$ ) also confirms laminar flow. (The transition to turbulence in external flows typically sets in at values of the Reynolds numbers of about $2-5$ $\times 10^{5}$.) Interestingly, the maxima in the local Reynolds number in Figs. 9(a) and 9(b) both establish very early in the liquid deformation process, prior to the onset of self-similar growth. The early rapid increase in $\mathrm{Re}_{\text {apex }}^{\max }$ followed by a monotonic decrease is due to the fact that the decrease in $R_{\text {apex }}$ with time occurs more rapidly than the corresponding increase in $V_{\text {apex }}$.

\section{B. Conic tip behavior quantified by the local Reynolds number}

Shown in Fig. 9(c) is the maximum apex Reynolds number achieved during each run for $0.1 \leq \mathrm{Re} \leq 50000$ at $\mathrm{Ca}=7.0834$ where $\operatorname{Re}_{\text {apex }}^{\text {max }}=\operatorname{Re} V_{\text {apex }}^{\max } R_{\text {apex }}^{\max }$. The quantities $R_{\text {apex }}^{\text {max }}$ and $V_{\text {apex }}^{\text {max }}$ denote the corresponding values of the tip curvature radius and tip speed. The function $\mathrm{Re}_{\text {apex }}^{\max }$ increases smoothly and monotonically with increasing $\operatorname{Re}$ such that $\operatorname{Re}_{\text {apex }}^{\max } \approx 0.1 \times \operatorname{Re}$ for $\operatorname{Re}>10$. It is evident from Fig. 9(c) that at intermediate and large values of $R e$, where the dominant opposing stresses are the Maxwell and capillary stress, the velocity and curvature radius track each other closely in magnitude. This is expected since the Maxwell pressure is always the dominant 

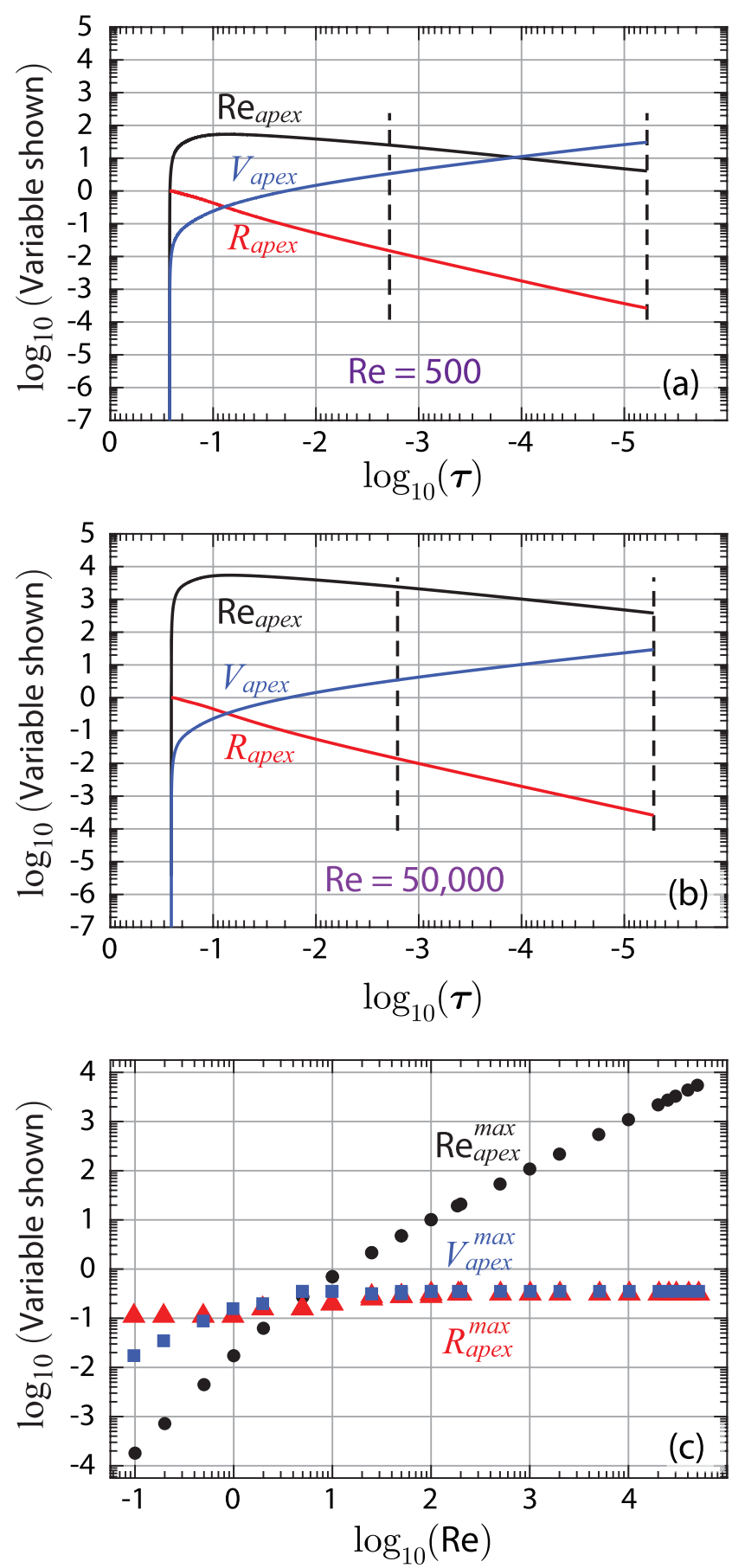

FIG. 9. [(a) and (b)] Double logarithmic plots showing apex curvature, apex flow speed, and apex (local) Reynolds number $\operatorname{Re}_{\text {apex }}=V_{\text {apex }} R_{\text {apex }} \times \operatorname{Re} v s=T_{\mathrm{B}}-T$ for $\mathrm{Re}=500$ and 50000 at $\mathrm{Ca}=7.0834$. Dashed vertical lines indicate the interval of self-similar growth, as designated in Fig. 7. (c) Double logarithmic plot showing the maximum apex Reynolds number achieved during each run for $0.1 \leq \mathrm{Re}$ $\leq 50000$ at $\mathrm{Ca}=7.0834$, where $\operatorname{Re}_{a p e x}^{\max }=\operatorname{Re} V_{a p e x}^{\max } R_{a p e x}^{\max } \cdot R_{a p e x}^{\max }$ and $V_{a p e x}^{\max }$ denote the corresponding values of the apex curvature radius and apex vertical speed. driving force and its magnitude is set by the apex curvature radius. We note that both $V_{\text {apex }}^{\max }$ and $R_{\text {apex }}^{\max }$ are rather independent of $\mathrm{Re}$ except in the viscous dominated regime at very small values of Re. This behavior confirms that these two quantities are governed by local conditions at the apex, where the electric field strength and curvature radius undergo self-enhancement and self-sharpening. This behavior stands in contrast to that at small values of the Reynolds number $\log _{10}(R e)<1$, where the dominant opposing stresses are the Maxwell and viscous normal stress. In particular, viscous normal stresses relay on longer range interactions mediated by liquid viscosity, which introduces dependence on Re. Close inspection of the curve $\mathrm{Re}_{\text {apex }}^{\max }$ in Fig. 9(c) reveals a change in slope at a value of about $\log _{10}(R e)=1$. This break in slope distinguishes viscous dominated behavior to the left where $\operatorname{Re}_{\text {apex }}^{\max } \sim \mathrm{Re}^{2}$ from the inertia dominated behavior to the right where $\operatorname{Re}_{\text {apex }}^{\max } \sim \operatorname{Re}$.

\section{Experimental restrictions on liquid film thickness and electric field strength}

\section{Restrictions due to Bond number}

In this study, the capillary number was held fixed at a value $\mathrm{Ca}=7.0834$ and gravity was neglected by requiring that $\mathrm{Bo}$ $=\rho g h_{o}^{2} / \gamma<<1$. The simulations were also terminated once the dimensionless apex radius of curvature (normalized by $h_{o}$ ) attained the value $2.722 \times 10^{-4}$. These constraints therefore enforce certain requirements on physical systems that may be used to test the predictions reported here. In comparing results with future experiments, it is also important that electric field values at the apex not exceed values for field ion emission in liquid metals typically of order $10^{10} \mathrm{~V} / \mathrm{m},{ }^{11}$ though the actual values depend not only on the liquid metal but also the operating temperature and vacuum conditions.

\section{Restrictions due to maximum field strength}

To ascertain the parameter range of validity for the simulations in this study, we provide in Table III a list of derived values for three liquid metals at their melting temperature commonly used in LMIS systems, namely, gallium (Ga), indium (In), and cesium (Cs). The entries shown were computed as follows. From the scalings in Table I, a fixed value of $R e$ for a given liquid corresponds to a specific initial voltage potential $\phi_{o}$. The constraint $\mathrm{Ca}=7.0834$ then establishes the corresponding thickness $h_{o}$ of the initial liquid layer, which in turn sets the initial field strength value $E_{o}=\phi_{o} / h_{o}$. The material constants and the value $h_{o}$ then set the corresponding value of the Bond number. For ground based experimental studies, it would be necessary to satisfy the inequality Bo $\ll 1$ when making comparison to the findings of our study. Of course, one can always redo our numerical study using a larger value of $\mathrm{Ca}$, which for a given value of $\phi_{o}$ would ensure a smaller value of $h_{o}$ and therefore a smaller Bond number. Too large a value of $\mathrm{Ca}$, however, can trigger surface waves leading to multiple protrusions and interference effects.

Another important consideration for experimentalists is the small time window for measurement before onset of ion emission, a phenomenon that has not yet been incorporated into numerical models probing self-similarity in electrified liquids. The challenge in incorporating that phenomenon stems from the disparate length and time scales characterizing the hydrodynamic flow and field 
TABLE III. Material constants and operating values for three liquid metals-Ga, In, and Cs-often used in LMIS systems. Ga values from Ref. 24; In and Cs values from Ref. 40. Quantities $\gamma, \mu$, and $\rho$ denote, respectively, the liquid surface tension, viscosity, and density evaluated at the melting point. Definitions for Re, Ca, and Bo are given in Table I. Remaining quantities refer to the characteristic quantities defined in Table I given by the voltage potential $\phi_{0}$, the system size $h_{0}$, and the initial electric field strength $E_{0}$.

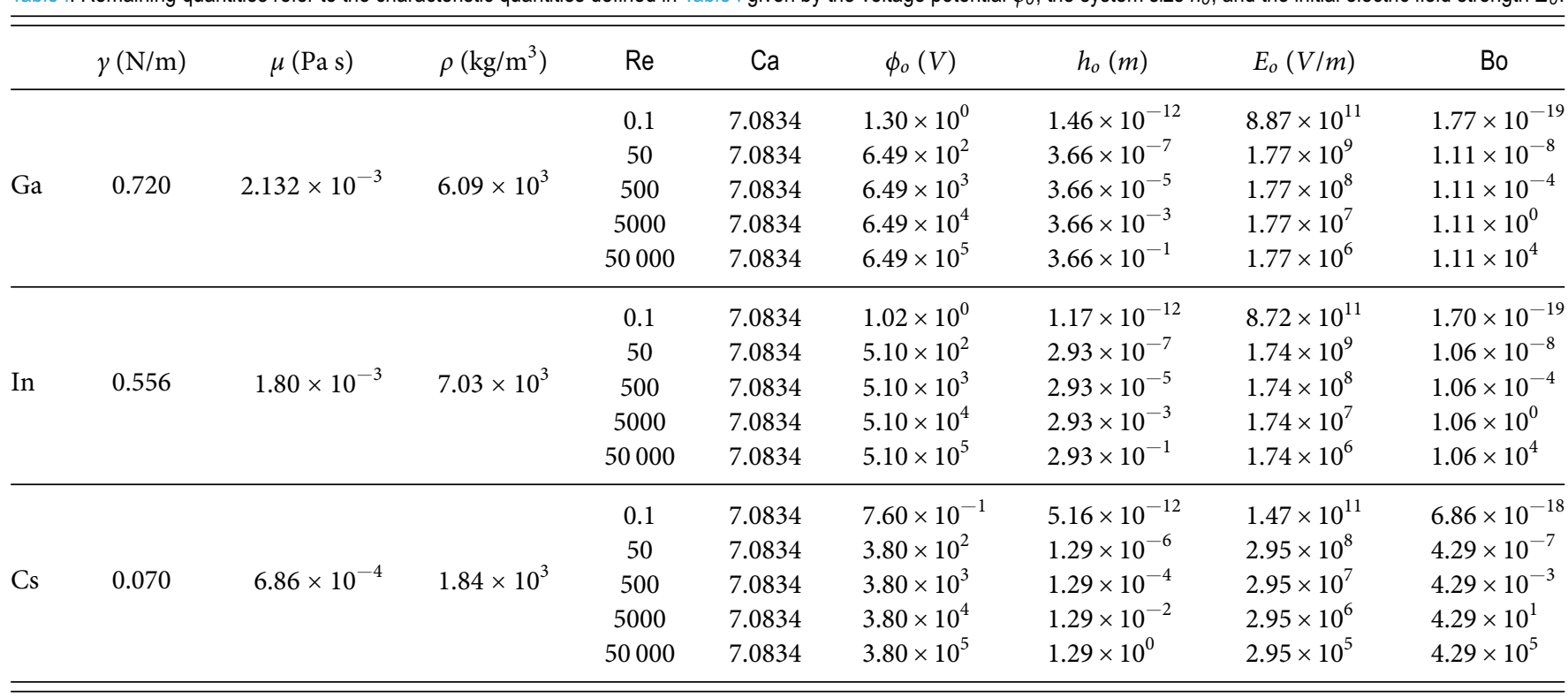

evaporation and related processes. Be that as it may, the initial field strength value in simulations should therefore not exceed field emission values. Shown in Table III are values for the initial electric field strengths $E_{o}$ for the specified values of $\mathrm{Re}$ and $\mathrm{Ca}$ used in our simulations as determined from the definitions in Table I. The values of $E_{o}$ for the smallest Reynolds number simulated in our study, namely, $\operatorname{Re}=0.1$, would likely exceed the limit for ion emission; the corresponding values of $h_{o}$ are also unrealistically small. For the material constants listed, measurements at very low Reynolds numbers are likely not possible even at different values of $\mathrm{Ca}$. According to the expressions for $\mathrm{Re}$ and $\mathrm{Ca}$ given in Table $\mathrm{I}$, a fixed value of $\operatorname{Re}$ corresponds to a fixed value of the voltage potential $\phi_{0}$. Therefore, smaller values of $\mathrm{Ca}$ correspond to larger values of $h_{o}$ and ultimately smaller values of the field strength $E_{o}=\phi_{o} / h_{o}$. For $\operatorname{Re}$ $=0.1$, the value $E_{o}$ would have to be reduced by about two orders of magnitude to forestall ion emission, which could be accomplished by increasing $h_{o}$ by two orders of magnitude. However, that would still lead to unrealistically small values of $h_{o}$, in the range of $0.1 \mathrm{~nm}$. It is therefore unlikely that self-similar behavior in the Stokes regime can be tested in vacuum using the model geometry depicted in Fig. 2.

\section{RECENT EXPERIMENTS OF CONIC CUSP GROWTH IN PARTIALLY CONDUCTING AND LEAKY DIELECTRIC LIQUIDS}

Due to the high flow speeds typical of conic cusp growth in liquids, some experimentalists have tried to slow the growth process by using partially conducting fluids beneath a layer of insulating liquid. This has allowed direct optical tracking and quantification of cuspidal acceleration undergoing self-similar growth. Oddershede and Nagel ${ }^{38}$ used high speed photography to track the evolution of a sharp liquid spout formed in response to a large normal electric field applied to a layer of distilled water containing $\mathrm{NaCl}$ beneath a layer of silicone oil. Above a critical field strength, the water generated a cuspidal shape whose apex curvature and overall height were shown to exhibit divergent self-similar growth extending almost three decades in time. The authors indicated that close to the blowup time, the shape of the conducting liquid tip was not exactly a cone but resembled more a square-root cusp. Simulations of the type described in this work could be expanded to examine such systems by replacing the perfectly conducting liquid with a partially conducting one and replacing the vacuum layer with a viscous liquid layer. These changes, of course, will modify the boundary conditions at the moving interface.

More recently, Elele et al. ${ }^{39}$ have been successful in monitoring the field driven deformation of submillimeter size sessile droplets into conical shapes under ambient conditions by limiting the buildup of electric charge along the free surface. This allowed a sufficiently long period of distortion and growth before the onset of electrospray, which was achieved by separating the liquid droplet from the ground electrode with an electrically insulating film that prevented electric current from flowing between opposing electrodes. They examined the behavior of a number of partially conducting and leaky dielectric liquids using both ground based and space based studies. Their microgravity experiments carried out in the International Space Station allowed measurements on larger droplet sizes in the centimeter range. Elele et al. confirmed the growth of conic tip shapes, exhibiting a wide range of internal vertex angles dependent on operating conditions and self-similar behavior in the gap distance between the liquid apex and counter electrode, found to scale roughly as $\tau^{-2 / 3}$. Although this behavior is suggestive of Zubarev's prediction, it is important to note that the liquids used in these experiments were not perfectly conducting nor 
could the flow be considered inviscidlike, two critical assumptions in Zubarev's analysis.

\section{CONCLUSION}

The numerical simulations described in this work demonstrate how the free surface of an electrified, perfectly conducting viscous liquid undergoes deformation toward a rapidly accelerating conic cusp whose tip exhibits self-similar growth for all Reynolds numbers spanning the viscous to inertial regime. Field enhancement caused by the continuous sharpening of the conic apex causes divergent power law growth in finite time. Temporal tracking of the interface shape, normal stresses, and forces per unit volume at the apex reveals how the blowup exponents vary with increasing $\mathrm{Re}$, made to span a dynamic range of six orders of magnitude. The asymptotic values of these exponents are in excellent agreement with previous predictions in the literature in two distinguished limits, namely, $\mathrm{Re}=0$ (Stokes regime) and $\mathrm{Re}=\infty$ (inviscid regime). The simulation results support the conclusion that at very small Reynolds numbers, the dominant competition in the forces per unit volume acting on the conic apex is represented by the Maxwell and viscous normal stress, while at intermediate and higher Reynolds numbers, the dominant competition transitions to the Maxwell and capillary stress.

The temporal behavior of the terms in the normal stress boundary condition at the moving interface indicates that for Reynolds numbers higher than about 50 , the viscous normal stress is rather insignificant in comparison with the Maxwell and the capillary pressure. Similar tracking of the terms in the Navier-Stokes equation, however, reveals that viscous normal forces per unit volume acting at the conic apex cannot be neglected for values of Re below about $3 \times 10^{4}$ since they remain comparable in magnitude to the apical pressure gradient and apical inertial forces. Previous studies of dynamic cone growth based on Bernoulli's equation have tended to neglect altogether the role of viscous stresses in the cone tip.

We also note that Zubarev's ${ }^{20}$ original prediction for selfsimilar growth in the inviscid limit led to power law exponents characterized by a ratio of two integers. In particular, he reported that the capillary pressure, the Maxwell pressure, and the kinetic energy per unit volume all scaled as $\tau^{-2 / 3}$. By contrast, Fontelos et al. ${ }^{34}$ uncovered that in the Stokes regime, the corresponding exponents are not simple integer ratios but instead depend functionally on the cone interior half-angle. Our study, which reveals the range and behavior of exponents characterizing the forces acting at the conic apex, confirms that the exponents at finite Re are never simple integer values, which reflects the fact that the forces at the tip depend sensitively on the slope of the dynamic cone as the system approaches the singular blowup point.

\section{ACKNOWLEDGMENTS}

The authors gratefully acknowledge financial support from a 2014 NASA Space Technology Research Fellowship (T.G.A.) and the NASA/Jet Propulsion Laboratory (JPL) President's and Director's Fund (S.M.T.). T.G.A. also wishes to thank Andy Ylitalo (2016 Caltech Summer Undergraduate Research Fellow) for assistance with the numerical simulations during early stages of this work and fellow office mate Chengzhe Zhou for helpful and interesting discussions about electrified liquid behavior.

\section{REFERENCES}

${ }^{1}$ J. Larmor, “The influence of electrification on ripples," Proc. Cambridge Philos. Soc. 7, 69-71 (1890).

${ }^{2}$ L. Tonks, "A theory of liquid surface rupture by a uniform electric field," Phys. Rev 48, 562-568 (1935).

${ }^{3}$ Y. L. Frenkel, "On the Tonks theory of liquid surface rupture by a constant electric field in vacuo,” Zh. Eksp. Teor. Fiz. 6, 348-350 (1936).

${ }^{4}$ V. E. Krohn, "Liquid metal droplets for heavy particle propulsion," Prog. Astronaut. Aeronaut. 5, 73-80 (1961).

${ }^{5}$ J. Perel, A. Y. Yahiku, J. F. Mahoney, H. L. Daley, and A. Sherman, "Operational characteristics of colloid thrusters,” J. Spacecr. Rockets 8, 702-708 (1971).

${ }^{6}$ A. G. Bailey, J. E. Bracher, and H. J. Von Rohden, "A capillary-fed annular colloid thruster," J. Spacecr. Rockets 9, 518-521 (1972).

${ }^{7}$ S. Zafran, J. C. Beynon, P. W. Kidd, H. Shelton, and F. A. Jackson, “One-mlb colloid thruster system development,” J. Spacecr. Rockets 10, 531-533 (1973).

${ }^{8}$ M. N. Huberman and S. G. Rosen, "Advanced high-thrust colloid sources," J. Spacecr. Rockets 11, 475-480 (1974).

${ }^{9}$ V. E. Krohn and G. R. Ringo, "Secondary-ion collection system for an ion microprobe analyzer of high mass resolution," Rev. Sci. Instrum. 43, 1771-1772 (1972).

${ }^{10}$ R. L. Seliger, J. W. Ward, V. Wang, and R. L. Kubena, “A high-intensity scanning ion probe with submicrometer spot size," Appl. Phys. Lett. 34, 310-312 (1979).

${ }^{11}$ R. G. Forbes and G. L. R. Mair, "Liquid metal ion sources," in Handbook of Charged Particle Optics, 2nd ed., edited by J. Orloff (CRC Press, Boca Raton, FL, 2008), Chap. 2.

${ }^{12}$ W. P. Wright and P. Ferrer, "Electric micropropulsion systems," Prog. Aerosp. Sci. 74, 48-61 (2015).

${ }^{13}$ M. K. Bharti and S. Chalia, "Literature study of field emission electric propulsion microthruster," Int. Res. J. Eng. Tech. 4, 2777-2781 (2017).

${ }^{14}$ NASA Technology Roadmaps TA2: In-Space Propulsion Technologies, www.nasa.gov/offices/oct/home/roadmaps/index.html, 2015.

${ }^{15}$ Z. You, Space Microsystems and Micro/Nano Satellites (National Defense Industry Press; Butterworth-Heinemann, Oxford, UK, 2017).

${ }^{16}$ Lord Rayleigh, "On the equilibrium of liquid conducting masses charged with electricity,” Philos. Mag. 14, 184-186 (1882).

${ }^{17}$ G. I. Taylor, "Disintegration of water drops in an electric field," Proc. R. Soc. London, Ser. A 280, 383-397 (1964).

${ }^{18}$ W. Gilbert, de Magnete (Dover, New York, 1958), Vol. II, Chap. II, p. 89.

${ }^{19}$ S. Gray, “A letter concerning the electricity of water," Philos. Trans. R. Soc. London 37, 227-230 (1731).

${ }^{20}$ N. M. Zubarev, "Formation of conic cusps at the surface of liquid metal in electric field,” J. Exp. Theor. Phys. Lett. 73, 544-548 (2001).

${ }^{21}$ V. G. Suvorov and E. A. Litvinov, "Dynamic Taylor cone formation on liquid metal surface: Numerical modeling," J. Phys. D: Appl. Phys. 33, 1245-1251 (2000).

${ }^{22}$ O. M. Belozerkovskii, Numerical Simulations in Continuous Media Mechanics (Physics and Mathematics Literature, Moscow, 1994), p. 442.

${ }^{23} \mathrm{C}$. Zheng and T. Linsu, "A new approach to simulating the operation of liquid metal ion sources,” J. Vac. Sci. Technol., B 6, 2104-2107 (1988).

${ }^{24}$ V. G. Suvorov, "Numerical analysis of liquid metal flow in the presence of an electric field: Application to liquid metal ion source," Surf. Interface Anal. 36, 421425 (2004).

${ }^{25}$ V. G. Suvorov and N. M. Zubarev, "Formation of the Taylor cone on the surface of liquid metal in the presence of an electric field," J. Phys. D: Appl. Phys. 37, 289-297 (2004).

${ }^{26}$ R. T. Collins, J. J. Jones, M. T. Harris, and O. A. Basaran, "Electrohydrodynamic tip streaming and emission of charged drops from liquid cones," Nat. Phys. 4, 149-154 (2008).

${ }^{27}$ R. T. Collins, K. Sambath, M. T. Harris, and O. A. Basaran, "Universal scaling laws for the disintegration of electrified drops," Proc. Natl. Acad. Sci. U. S. A. 110, 4905-4910 (2013). 
${ }^{28} \mathrm{~J}$. T. Holgate and M. Coppins, "Shapes, stability, and hysteresis of rotating and charged axisymmetric drops in a vacuum," Phys. Fluids 30, 064107 (2018).

${ }^{29}$ J. F. de la Mora and I. G. Loscertales, "The current emitted by highly conducting Taylor cones,” J. Fluid Mech. 260, 155-184 (1994).

${ }^{30}$ A. M. Gañán-Calvo, "Cone-jet analytical extension of Taylor’s electrostatic solution and the asymptotic universal scaling laws in electrospraying," Phys. Rev. Lett. 79, 217-220 (1997).

${ }^{31}$ J. C. Burton and P. Taborek, "Simulations of coulombic fission of charged inviscid drops,” Phys. Rev. Lett. 106, 144501 (2011).

${ }^{32} \mathrm{M}$. Garzon, L. J. Gray, and J. A. Sethian, "Numerical simulations of electrostatically driven jets from nonviscous droplets," Phys. Rev. E 89, 033011 (2014).

${ }^{33}$ S. I. Betelú, M. A. Fontelos, U. Kindelán, and O. Vantzos, "Singularities on charged viscous droplets," Phys. Fluids 18, 051706 (2006).
${ }^{34} \mathrm{M}$. A. Fontelos, U. Kindelán, and O. Vantzos, "Evolution of neutral and charged droplets in an electric field," Phys. Fluids 20, 092110 (2008).

${ }^{35}$ We note a typographical error in Eq. (7) of Ref. 34 where the quantity $\gamma \mathcal{K}$ should read $-2 \gamma \mathcal{K}$.

${ }^{36}$ COMSOL Multiphysics, Inc., V5.2a, Multifluidics module, Burlington, MA.

${ }^{37}$ L. G. Leal, Advanced Transport Phenomena: Fluid Mechanics and Convective Transport Processes (Cambridge University Press, 2007).

${ }^{38}$ L. Oddershede and S. R. Nagel, "Singularity during the onset of an electrohydrodynamic spout," Phys. Rev. Lett. 85, 1234-1237 (2000).

${ }^{39}$ E. O. Elele, Y. Shen, D. R. Pettit, and B. Khusid, "Detection of a dynamic coneshaped meniscus on the surface of fluids in electric fields," Phys. Rev. Lett. 114, 054501 (2015).

${ }^{40}$ T. Iida and R. I. L. Guthrie, The Physical Properties of Liquid Metals (Oxford University Press, 2007). 